\title{
DIATOMIC GAS DIFFUSING IN A BACKGROUND MEDIUM: KINETIC APPROACH AND REACTION-DIFFUSION EQUATIONS*
}

\author{
MARZIA BISI ${ }^{\dagger}$ AND GIAMPIERO SPIGA ${ }^{\ddagger}$
}

\begin{abstract}
The problem of a diatomic gas made up by atoms and molecules undergoing reactions of dissociation and recombination through a transition state is addressed. The gas is diffusing in a scattering and dissociating background medium. Extended Boltzmann-like kinetic equations for the relevant distribution functions and exact balance equations for mass, momentum, and energy are derived and discussed. It is shown that, in the asymptotic limit of small Knudsen numbers, a suitable scaling leads to a consistent formal derivation of hydrodynamic equations of reaction-diffusion type for number densities of atoms and molecules.
\end{abstract}

Key words. Kinetic theory, Reactions of dissociation/recombination, Asymptotic limit, Reaction-diffusion equations.

Subject classifications. $82 \mathrm{C} 40,76 \mathrm{P} 05,35 \mathrm{~K} 57$

\section{Introduction}

There exists an ample literature about the uneasy problem of a consistent quantitative description of chemically reacting rarefied flows starting from a kinetic approach. We quote, for instance, and among many others, the books $[9,5]$ and references therein, and some recent contributions [6, 10, 8, 20, 15, 12]. Most of the literature on the subject deals with bimolecular reversible or irreversible reactions, which exclude some very important and common reactive processes, like reactions of dissociation and recombination, one of the essential ingredients characterizing diatomic gases in the air. This is the problem that will be dealt with here, following the scheme of [11], where kinetic equations were derived and discussed for a single diatomic gas in the frame of the so-called transition-state theory [18]. It has been possible to obtain explicitly conservation laws, collision equilibria, and fluid-dynamic equations of the Euler type for the main macroscopic fields. We aim at extending such an approach to the case of potential practical application, and of mathematical and physical interest, in which the dilute diatomic gas is not an isolated system, but its atoms and molecules also interact with a background medium in which they are embedded, exchanging momentum and energy by elastic collisions, and undergoing chemical reactions with field particles as well. After deriving kinetic equations for the participating distribution functions, and exact non-closed balance equations for the observable fields, we analyze the hydrodynamic limit in the collision dominated regime, and show that suitable scalings at the mesoscopic level lead to an explicit formal derivation of a closed set of fluid-dynamic equations, which turn out to be of reaction-diffusion type, similar to, but of course different from, those which have been recently derived for various scenarios of gas mixtures with bimolecular reactions [1].

According to the model proposed in the paper [11], briefly recalled here for the readers' convenience, a diatomic gas can be described as a mixture of three interacting species: atoms $A$ (with mass $m_{1}$ ), stable diatomic molecules $A_{2}$ (with mass $m_{2}=2 m_{1}$ ), and unstable molecules $A_{2}^{*}$ (with mass $m_{3}=m_{2}$ ) which play the role of a

\footnotetext{
${ }^{*}$ Received: May 30, 2006; accepted (in revised version): October 10, 2006. Communicated by Lorenzo Pareschi.

${ }^{\dagger}$ Dipartimento di Matematica, Università di Parma, Viale G. P. Usberti 53/A, 43100 Parma, Italy (marzia.bisi@unipr.it).

‡Dipartimento di Matematica, Università di Parma, Viale G. P. Usberti 53/A, 43100 Parma, Italy (giampiero.spiga@unipr.it).
} 
transition state. For the purpose of a correct energy balance, it suffices to assume that stable and unstable molecules are endowed with their own internal energies, which are denoted, respectively, by $-Q$ and $E$, where $Q$ is a fixed positive quantity, while $E$ is a continuous variable ranging on the positive real axis. In addition, we extend now such scenario by assuming that the present rarefied mixture is diffusing in a much denser medium $B$, considered as a fixed background whose evolution is not influenced by collisions with atoms/molecules. In the sequel the background will be identified by the index 0 , and it will be assumed in local thermodynamical equilibrium, namely with distribution function $f_{0}=n_{0} M_{0}$, where $M_{0}$ stands for the normalized Maxwellian

$$
M_{0}=\left(\frac{m_{0}}{2 \pi K T_{0}}\right)^{\frac{3}{2}} \exp \left(-\frac{m_{0}}{2 K T_{0}}\left(\mathbf{v}-\mathbf{u}_{0}\right)^{2}\right),
$$

and $m_{0}, n_{0}, \mathbf{u}_{0}, T_{0}$ denote, respectively, the background particle mass, number density, drift velocity, and temperature. Analogously, fields corresponding to the species $A$, $A_{2}, A_{2}^{*}$ will be labelled by the indices $1,2,3$, respectively.

Both atoms $A$ and stable molecules $A_{2}$ may be involved in binary reversible elastic collisions with other atoms, stable molecules and background particles:

$$
\begin{aligned}
& \text { (E1) } A+P \quad \leftrightarrow A+P, \\
& \text { (E2) } A_{2}+P \quad \leftrightarrow \quad A_{2}+P,
\end{aligned}
$$

where $P=A, A_{2}, B$. Unstable molecules $A_{2}^{*}$ are not subjected to elastic scattering, since they are characterized by a very small mean lifetime, so that once an unstable molecule is created, it easily disappears through a (fast) chemical reaction. In our model we assume that two atoms $A$ may form a stable molecule $A_{2}$ passing through the transition state $A_{2}^{*}$, while, on the other hand, both stable and unstable diatomic molecules may dissociate into two atoms. More precisely, the recombination process occurs in two steps:

$$
\begin{aligned}
& (R) A+A \rightarrow A_{2}^{*}, \\
& (I) A_{2}^{*}+P \quad \rightarrow A_{2}+P,
\end{aligned}
$$

where $P=A, A_{2}, B$. The reaction $(R)$ may occur only if the relative speed of the incoming particles fulfills the upper and lower energy thresholds determined by the internal energy $E$ of $A_{2}^{*}$ [11]; otherwise, only an elastic scattering may occur. The second step $(I)$ may be considered as an inelastic scattering process that causes the de-excitation of $A_{2}^{*}$. Dissociation occurs via two possible reactions:

$$
\begin{aligned}
& (D 1) A_{2}+P \rightarrow 2 A+P, \\
& (D 2) A_{2}^{*}+P \rightarrow 2 A+P .
\end{aligned}
$$

The conservation of energy determines an energy threshold for $(D 1)$ (it is an endothermic process), while for (D2), being exothermic, no threshold is needed [11].

The paper is organized as follows. A mesoscopic description of the preceding physical situation is worked out in the next Section in the frame of a suitable formulation of the Boltzmann equation [3], and the relevant extended kinetic equations are written down and discussed in terms of collision frequencies and transition probabilities. A huge number of such microscopic quantities appears, of course, and the task of a realistic expression for all of them is quite hopeless. However, as pointed out in [11], it is remarkable that several crucial mathematical and physical features of 
the problem are determined solely by the conservation properties that they have to fulfill because of their own definition, and independently from their explicit form. For instance, based only on the knowledge of conservation laws and basic properties of microscopic quantities, exact non-closed balance equations are derived for the macroscopic fields of physical interest in Section 3. They constitute a set of five partial differential equations quantifying mass conservation and exchange of momentum and energy with the background, due to both elastic scattering and chemical reactions, and retain integral non-local terms which describe binary collisions between test and field particles. A deeper analysis of the present evolution equations at kinetic level is in progress, and will be matter of future work, concerning collision equilibria, $\mathrm{H}$ theorem, hydrodynamic limits. The approach (restricted clearly to a formal level, a rigorous treatment seems premature at this stage) is technically heavy and uneasy because of the several interaction mechanisms involved and relevant collision operators. We focus here, among the above open problems, on existence of physical regimes in which dominant processes lead to a consistent macroscopic closure of reactiondiffusion type, moving, with the necessary extensions and modifications, along the lines of Ref. [1], where this kind of derivation first appeared in the literature (for a simpler scenario). Indeed, Section 4 deals with the asymptotic analysis for small mean paths (times) of the previous kinetic equations. The investigation is restricted in this first approach to a Maxwellian collision model, which allows as usual a fully explicit analytical manipulation. Of course several small parameters can show up after adimensionalization, according to the several physical phenomena involved in the process. It is shown that a suitable scaling yields a consistent formal derivation of a closed set of reaction-diffusion equations for the densities of the two stable species, with reactive contributions that are rational functions of the densities themselves, and diffusion coefficients depending on elastic collision frequencies and background temperature. Analysis of other meaningful scalings, as well as of other possible limits, is also scheduled as future work.

\section{Reactions of recombination/dissociation}

By means of usual methods of extended kinetic theory [4, 13], we can write down kinetic equations for the evolution of the distribution functions of the species $A, A_{2}$, $A_{2}^{*}$, denoted by $f_{1}(\mathbf{v}), f_{2}(\mathbf{v}), \varphi_{3}(\mathbf{v}, E)$, respectively:

$$
\begin{aligned}
\frac{\partial f_{1}}{\partial t}+\mathbf{v} \cdot \frac{\partial f_{1}}{\partial \mathbf{x}}= & \mathcal{Q}_{10}\left(f_{1}, f_{0}\right)+\mathcal{Q}_{11}\left(f_{1}\right)+\mathcal{Q}_{12}\left(f_{1}, f_{2}\right) \\
& +\mathcal{J}_{1}^{*}\left(f_{1}, f_{2}, \varphi_{3}\right)+\mathcal{J}_{1}^{d}\left(f_{1}, f_{2}\right)+\mathcal{J}_{1}^{r}\left(f_{1}\right)+\mathcal{I}_{1}^{*}\left(\varphi_{3}, f_{0}\right)+\mathcal{I}_{1}^{d}\left(f_{2}, f_{0}\right), \\
\frac{\partial f_{2}}{\partial t}+\mathbf{v} \cdot \frac{\partial f_{2}}{\partial \mathbf{x}}= & \mathcal{Q}_{20}\left(f_{2}, f_{0}\right)+\mathcal{Q}_{21}\left(f_{2}, f_{1}\right)+\mathcal{Q}_{22}\left(f_{2}\right) \\
& +\mathcal{J}_{2}^{*}\left(f_{1}, f_{2}, \varphi_{3}\right)+\mathcal{J}_{2}^{d}\left(f_{1}, f_{2}\right)+\mathcal{I}_{2}^{*}\left(\varphi_{3}, f_{0}\right)+\mathcal{I}_{2}^{d}\left(f_{2}, f_{0}\right), \\
\frac{\partial \varphi_{3}}{\partial t}+\mathbf{v} \cdot \frac{\partial \varphi_{3}}{\partial \mathbf{x}}= & \mathcal{J}_{3}^{*}\left(f_{1}, f_{2}, \varphi_{3}\right)+\mathcal{J}_{3}^{r}\left(f_{1}\right)+\mathcal{I}_{3}^{*}\left(\varphi_{3}, f_{0}\right) .
\end{aligned}
$$

Note that the distribution $\varphi_{3}$ for the unstable molecules depends also on the internal energy variable $E$; the usual velocity distribution is recovered as

$$
f_{3}(\mathbf{v})=\int \varphi_{3}(\mathbf{v}, E) d E .
$$

In (2.1), symbols $\mathcal{Q}_{i j}$ stand for elastic scattering operators, $\mathcal{J}_{i}$ denote chemical operators relevant to the reactions not involving the background, while $\mathcal{I}_{i}$ identify chemical 
terms involving the background. The star superscript affects operators involving as reactants the unstable molecules $A_{2}^{*}$, while remaining chemical operators show $d$ or $r$ superscript to distinguish if they are relevant to the dissociation or to the recombination process.

Bi-species elastic collision terms appearing in (2.1) are well known in classical kinetic theory [4], and we will not enter in the details of the derivation:

$$
\mathcal{Q}_{i j}(\mathbf{v})=\int_{\mathbb{R}^{3}} \int_{S^{2}} b_{i j}(\mathbf{v}-\mathbf{w}, \hat{\mathbf{n}})\left[f_{i}(\tilde{\mathbf{v}}) f_{j}(\tilde{\mathbf{w}})-f_{i}(\mathbf{v}) f_{j}(\mathbf{w})\right] d \mathbf{w} d \hat{\mathbf{n}}
$$

for each pair $(i, j)=(1,0),(1,1),(1,2),(2,0),(2,1),(2,2)$. The function $b_{i j}$ represents the microscopic differential collision frequency of the elastic encounter $(i, j)$. Thanks to conservation of momentum and kinetic energy by elastic scattering, post-collision velocities $(\tilde{\mathbf{v}}, \tilde{\mathbf{w}})$ can be expressed in terms of the incoming velocities $(\mathbf{v}, \mathbf{w})$ and of the unit vector $\hat{\mathbf{n}}$ (apse line) as

$$
\begin{aligned}
\tilde{\mathbf{v}} & =\mathbf{v}-\frac{2 m_{j}}{m_{i}+m_{j}}[(\mathbf{v}-\mathbf{w}) \cdot \hat{\mathbf{n}}] \hat{\mathbf{n}} \\
\tilde{\mathbf{w}} & =\mathbf{w}+\frac{2 m_{i}}{m_{i}+m_{j}}[(\mathbf{v}-\mathbf{w}) \cdot \hat{\mathbf{n}}] \hat{\mathbf{n}} .
\end{aligned}
$$

A similar treatment could be in order for bimolecular chemical reactions, as extensively discussed in $[14,8]$. However, we will describe these reactive collisions in terms of an equivalent scattering kernel formulation of the Boltzmann equation [3]. Chemical operators will involve total microscopic collision frequencies $g_{i j}^{\mathrm{k}}$, and scattering probability distributions of the kind $\Pi_{i j}^{h, \mathrm{k}}$. The superscript $\mathrm{k}$ shall be $\mathrm{k}=d, r, i$ correspondingly to dissociations $(D 1),(D 2)$, recombination $(R)$ and inelastic scattering $(I)$ respectively. The other superscript $h$ denotes the species of the considered outgoing particle: for instance, $\Pi_{30}^{2, i}\left(\mathbf{v}^{\prime}, E ; \mathbf{w}^{\prime} \rightarrow \mathbf{v}\right)$ represents the probability density that the outcoming particle 2 attains velocity $\mathbf{v}$ as a result of a de-excitation collision of a particle 3 with velocity $\mathbf{v}^{\prime}$ and internal energy $E$, against a background particle with velocity $\mathbf{w}^{\prime}$. The explicit expressions of the scattering kernels, involving necessarily also delta functions, may be deduced from the appropriate transition probabilities, and satisfy all obvious indistinguishableness constraints. We are interested here in deriving consistently as much practical information as possible from their basic properties, independently from their explicit expression. Operators $\mathcal{J}_{i}$ appearing in the Boltzmann equations (2.1) have been introduced and extensively discussed in [11]. In the evolution equation for atoms $A$ we have

$$
\begin{aligned}
& \mathcal{J}_{1}^{*}\left(f_{1}, f_{2}, \varphi_{3}\right)= \\
= & 3 \iiint g_{13}^{d}\left(\left|\mathbf{v}^{\prime}-\mathbf{w}^{\prime}\right|, E\right) \Pi_{13}^{1, d}\left(\mathbf{v}^{\prime} ; \mathbf{w}^{\prime}, E \rightarrow \mathbf{v}\right) f_{1}\left(\mathbf{v}^{\prime}\right) \varphi_{3}\left(\mathbf{w}^{\prime}, E\right) d E d \mathbf{v}^{\prime} d \mathbf{w}^{\prime} \\
& -f_{1}(\mathbf{v}) \iint g_{13}^{d}(|\mathbf{v}-\mathbf{w}|, E) \varphi_{3}(\mathbf{w}, E) d E d \mathbf{w} \\
& +2 \iiint g_{32}^{d}\left(\left|\mathbf{v}^{\prime}-\mathbf{w}^{\prime}\right|, E\right) \Pi_{32}^{1, d}\left(\mathbf{v}^{\prime}, E ; \mathbf{w}^{\prime} \rightarrow \mathbf{v}\right) \varphi_{3}\left(\mathbf{v}^{\prime}, E\right) f_{2}\left(\mathbf{w}^{\prime}\right) d E d \mathbf{v}^{\prime} d \mathbf{w}^{\prime} \\
& +\iiint g_{13}^{i}\left(\left|\mathbf{v}^{\prime}-\mathbf{w}^{\prime}\right|, E\right) \Pi_{13}^{1, i}\left(\mathbf{v}^{\prime} ; \mathbf{w}^{\prime}, E \rightarrow \mathbf{v}\right) f_{1}\left(\mathbf{v}^{\prime}\right) \varphi_{3}\left(\mathbf{w}^{\prime}, E\right) d E d \mathbf{v}^{\prime} d \mathbf{w}^{\prime} \\
& -f_{1}(\mathbf{v}) \iint g_{13}^{i}(|\mathbf{v}-\mathbf{w}|, E) \varphi_{3}(\mathbf{w}, E) d E d \mathbf{w},
\end{aligned}
$$




$$
\begin{aligned}
\mathcal{J}_{1}^{d}\left(f_{1}, f_{2}\right)= & 3 \iint g_{12}^{d}\left(\left|\mathbf{v}^{\prime}-\mathbf{w}^{\prime}\right|\right) \Pi_{12}^{1, d}\left(\mathbf{v}^{\prime} ; \mathbf{w}^{\prime} \rightarrow \mathbf{v}\right) f_{1}\left(\mathbf{v}^{\prime}\right) f_{2}\left(\mathbf{w}^{\prime}\right) d \mathbf{v}^{\prime} d \mathbf{w}^{\prime} \\
& -f_{1}(\mathbf{v}) \int g_{12}^{d}(|\mathbf{v}-\mathbf{w}|) f_{2}(\mathbf{w}) d \mathbf{w} \\
& +2 \iint g_{22}^{d}\left(\left|\mathbf{v}^{\prime}-\mathbf{w}^{\prime}\right|\right) \Pi_{22}^{1, d}\left(\mathbf{v}^{\prime} ; \mathbf{w}^{\prime} \rightarrow \mathbf{v}\right) f_{2}\left(\mathbf{v}^{\prime}\right) f_{2}\left(\mathbf{w}^{\prime}\right) d \mathbf{v}^{\prime} d \mathbf{w}^{\prime}, \\
& \mathcal{J}_{1}^{r}\left(f_{1}\right)=-2 f_{1}(\mathbf{v}) \int g_{11}^{r}(|\mathbf{v}-\mathbf{w}|) f_{1}(\mathbf{w}) d \mathbf{w} .
\end{aligned}
$$

Then, in the equation for stable molecules $A_{2}$ there appear

$$
\begin{gathered}
\mathcal{J}_{2}^{*}\left(f_{1}, f_{2}, \varphi_{3}\right)= \\
=\iiint g_{23}^{d}\left(\left|\mathbf{v}^{\prime}-\mathbf{w}^{\prime}\right|, E\right) \Pi_{23}^{2, d}\left(\mathbf{v}^{\prime} ; \mathbf{w}^{\prime}, E \rightarrow \mathbf{v}\right) f_{2}\left(\mathbf{v}^{\prime}\right) \varphi_{3}\left(\mathbf{w}^{\prime}, E\right) d E d \mathbf{v}^{\prime} d \mathbf{w}^{\prime} \\
-\quad f_{2}(\mathbf{v}) \iint g_{23}^{d}(|\mathbf{v}-\mathbf{w}|, E) \varphi_{3}(\mathbf{w}, E) d E d \mathbf{w} \\
+\iiint g_{31}^{i}\left(\left|\mathbf{v}^{\prime}-\mathbf{w}^{\prime}\right|, E\right) \Pi_{31}^{2, i}\left(\mathbf{v}^{\prime}, E ; \mathbf{w}^{\prime} \rightarrow \mathbf{v}\right) \varphi_{3}\left(\mathbf{v}^{\prime}, E\right) f_{1}\left(\mathbf{w}^{\prime}\right) d E d \mathbf{v}^{\prime} d \mathbf{w}^{\prime} \\
+\quad 2 \iiint g_{23}^{i}\left(\left|\mathbf{v}^{\prime}-\mathbf{w}^{\prime}\right|, E\right) \Pi_{23}^{2, i}\left(\mathbf{v}^{\prime} ; \mathbf{w}^{\prime}, E \rightarrow \mathbf{v}\right) f_{2}\left(\mathbf{v}^{\prime}\right) \varphi_{3}\left(\mathbf{w}^{\prime}, E\right) d E d \mathbf{v}^{\prime} d \mathbf{w}^{\prime} \\
-f_{2}(\mathbf{v}) \iint g_{23}^{i}(|\mathbf{v}-\mathbf{w}|, E) \varphi_{3}(\mathbf{w}, E) d E d \mathbf{w}, \\
\quad \mathcal{J}_{2}^{d}\left(f_{1}, f_{2}\right)=\iint g_{22}^{d}\left(\left|\mathbf{v}^{\prime}-\mathbf{w}^{\prime}\right|\right) \Pi_{22}^{2, d}\left(\mathbf{v}^{\prime} ; \mathbf{w}^{\prime} \rightarrow \mathbf{v}\right) f_{2}\left(\mathbf{v}^{\prime}\right) f_{2}\left(\mathbf{w}^{\prime}\right) d \mathbf{v}^{\prime} d \mathbf{w}^{\prime} \\
\quad-2 f_{2}(\mathbf{v}) \int g_{22}^{d}(|\mathbf{v}-\mathbf{w}|) f_{2}(\mathbf{w}) d \mathbf{w} \\
\quad-f_{2}(\mathbf{v}) \int g_{21}^{d}(|\mathbf{v}-\mathbf{w}|) f_{1}(\mathbf{w}) d \mathbf{w} .
\end{gathered}
$$

Finally, equation for unstable molecules $A_{2}^{*}$ involves

$$
\begin{aligned}
& \mathcal{J}_{3}^{*}\left(f_{1}, f_{2}, \varphi_{3}\right)=-\varphi_{3}(\mathbf{v}, E) \int\left[g_{31}^{d}(|\mathbf{v}-\mathbf{w}|, E)+g_{31}^{i}(|\mathbf{v}-\mathbf{w}|, E)\right] f_{1}(\mathbf{w}) d \mathbf{w} \\
&-\varphi_{3}(\mathbf{v}, E) \int\left[g_{32}^{d}(|\mathbf{v}-\mathbf{w}|, E)+g_{32}^{i}(|\mathbf{v}-\mathbf{w}|, E)\right] f_{2}(\mathbf{w}) d \mathbf{w}, \\
& \mathcal{J}_{3}^{r}\left(f_{1}\right)=\iint g_{11}^{r}\left(\left|\mathbf{v}^{\prime}-\mathbf{w}^{\prime}\right|\right) \Pi_{11}^{3, r}\left(\mathbf{v}^{\prime} ; \mathbf{w}^{\prime} \rightarrow \mathbf{v}, E\right) f_{1}\left(\mathbf{v}^{\prime}\right) f_{1}\left(\mathbf{w}^{\prime}\right) d \mathbf{v}^{\prime} d \mathbf{w}^{\prime} .
\end{aligned}
$$

Collision operators $\mathcal{I}_{i}$ may be derived by similar techniques, and take the form

$$
\mathcal{I}_{1}^{*}\left(\varphi_{3}, f_{0}\right)=2 \iiint g_{30}^{d}\left(\left|\mathbf{v}^{\prime}-\mathbf{w}^{\prime}\right|, E\right) \Pi_{30}^{1, d}\left(\mathbf{v}^{\prime}, E ; \mathbf{w}^{\prime} \rightarrow \mathbf{v}\right) \varphi_{3}\left(\mathbf{v}^{\prime}, E\right) f_{0}\left(\mathbf{w}^{\prime}\right) d E d \mathbf{v}^{\prime} d \mathbf{w}^{\prime},
$$




$$
\begin{gathered}
\mathcal{I}_{1}^{d}\left(f_{2}, f_{0}\right)=2 \iint g_{20}^{d}\left(\left|\mathbf{v}^{\prime}-\mathbf{w}^{\prime}\right|\right) \Pi_{20}^{1, d}\left(\mathbf{v}^{\prime} ; \mathbf{w}^{\prime} \rightarrow \mathbf{v}\right) f_{2}\left(\mathbf{v}^{\prime}\right) f_{0}\left(\mathbf{w}^{\prime}\right) d \mathbf{v}^{\prime} d \mathbf{w}^{\prime} \\
\mathcal{I}_{2}^{*}\left(\varphi_{3}, f_{0}\right)=\iiint g_{30}^{i}\left(\left|\mathbf{v}^{\prime}-\mathbf{w}^{\prime}\right|, E\right) \Pi_{30}^{2, i}\left(\mathbf{v}^{\prime}, E ; \mathbf{w}^{\prime} \rightarrow \mathbf{v}\right) \varphi_{3}\left(\mathbf{v}^{\prime}, E\right) f_{0}\left(\mathbf{w}^{\prime}\right) d E d \mathbf{v}^{\prime} d \mathbf{w}^{\prime} \\
\mathcal{I}_{2}^{d}\left(f_{2}, f_{0}\right)=-f_{2}(\mathbf{v}) \int g_{20}^{d}(|\mathbf{v}-\mathbf{w}|) f_{0}(\mathbf{w}) d \mathbf{w} \\
\mathcal{I}_{3}^{*}\left(\varphi_{3}, f_{0}\right)=-\varphi_{3}(\mathbf{v}, E) \int\left[g_{30}^{d}(|\mathbf{v}-\mathbf{w}|, E)+g_{30}^{i}(|\mathbf{v}-\mathbf{w}|, E)\right] f_{0}(\mathbf{w}) d \mathbf{w}
\end{gathered}
$$

\section{Exact macroscopic balance equations}

As well known in the literature [4], elastic collisions preserve mass, momentum and kinetic energy, hence elastic operators not involving the background medium satisfy the relations

$$
\begin{gathered}
\int \mathcal{Q}_{i j}(\mathbf{v}) d \mathbf{v}=0 \quad \forall i, j \quad \int \mathbf{v} \mathcal{Q}_{j j}(\mathbf{v}) d \mathbf{v}=\mathbf{0} \quad \forall j \quad \int v^{2} \mathcal{Q}_{j j}(\mathbf{v}) d \mathbf{v}=0 \quad \forall j \\
\int \mathbf{v}\left(\mathcal{Q}_{12}+2 \mathcal{Q}_{21}\right) d \mathbf{v}=\mathbf{0} \quad \int v^{2}\left(\mathcal{Q}_{12}+2 \mathcal{Q}_{21}\right) d \mathbf{v}=0
\end{gathered}
$$

As concerns linear elastic operators (describing scattering with the background) it is easy to check that the only collision invariant is the number density; by resorting to the pre-post-collision velocities relations (2.3), the exchange rates of momentum and energy with the host medium can be cast, for $j=1,2$, as [2]

$$
\begin{gathered}
\int \mathbf{v} \mathcal{Q}_{j 0}(\mathbf{v}) d \mathbf{v}=-\frac{2 m_{0}}{m_{j}+m_{0}} \iiint b_{j 0}(\mathbf{v}-\mathbf{w}, \hat{\mathbf{n}})[(\mathbf{v}-\mathbf{w}) \cdot \hat{\mathbf{n}}] \hat{\mathbf{n}} f_{j}(\mathbf{v}) f_{0}(\mathbf{w}) d \mathbf{v} d \mathbf{w} d \hat{\mathbf{n}}, \\
\int v^{2} \mathcal{Q}_{j 0}(\mathbf{v}) d \mathbf{v}=\frac{4 m_{0}}{m_{j}+m_{0}} \iiint b_{j 0}(\mathbf{v}-\mathbf{w}, \hat{\mathbf{n}})\left\{\frac{m_{0}}{m_{j}+m_{0}}[(\mathbf{v}-\mathbf{w}) \cdot \hat{\mathbf{n}}]^{2}\right. \\
-[(\mathbf{v}-\mathbf{w}) \cdot \hat{\mathbf{n}}](\mathbf{v} \cdot \hat{\mathbf{n}})\} f_{j}(\mathbf{v}) f_{0}(\mathbf{w}) d \mathbf{v} d \mathbf{w} d \hat{\mathbf{n}} .
\end{gathered}
$$

For chemical reactions, we have conservation (besides number of atoms) of momentum and total (kinetic plus internal) energy. For the recombination process $(R)$, such conservations read as

$$
\begin{array}{r}
m_{1}\left(\mathbf{v}^{\prime}+\mathbf{w}^{\prime}\right)=m_{3} \mathbf{v} \\
\frac{1}{2} m_{1}\left(\left(v^{\prime}\right)^{2}+\left(w^{\prime}\right)^{2}\right)=\frac{1}{2} m_{3} v^{2}+E
\end{array}
$$

so that, bearing in mind that $m_{3}=2 m_{1}$, the relevant collision kernel is explicitly given by

$$
\Pi_{11}^{3, r}\left(\mathbf{v}^{\prime} ; \mathbf{w}^{\prime} \rightarrow \mathbf{v}, E\right)=\delta\left(\frac{1}{2}\left(\mathbf{v}^{\prime}+\mathbf{w}^{\prime}\right)-\mathbf{v}\right) \delta\left(\frac{1}{4} m_{1}\left(\mathbf{v}^{\prime}-\mathbf{w}^{\prime}\right)^{2}-E\right)
$$


where $\delta$ is Dirac's delta distribution. Consequently the following conservation properties hold:

$$
\begin{aligned}
& \iint \Pi_{11}^{3, r}\left(\mathbf{v}^{\prime} ; \mathbf{w}^{\prime} \rightarrow \mathbf{v}, E\right) d E d \mathbf{v}=1, \\
& \iint 2 \mathbf{v} \Pi_{11}^{3, r}\left(\mathbf{v}^{\prime} ; \mathbf{w}^{\prime} \rightarrow \mathbf{v}, E\right) d E d \mathbf{v}=\mathbf{v}^{\prime}+\mathbf{w}^{\prime}, \\
& \iint\left(m_{1} v^{2}+E\right) \Pi_{11}^{3, r}\left(\mathbf{v}^{\prime} ; \mathbf{w}^{\prime} \rightarrow \mathbf{v}, E\right) d E d \mathbf{v}=\frac{1}{2} m_{1}\left(v^{\prime}\right)^{2}+\frac{1}{2} m_{1}\left(w^{\prime}\right)^{2} .
\end{aligned}
$$

Analogous considerations are in order for the reactive processes $(I),(D 1),(D 2)$, when the partner molecule $P=A, A_{2}$ (the option $P=B$ will be discussed later on in this section), even if in such cases the four conservation constraints do not allow to write down scattering kernels explicitly (there are more unknowns than conditions). Skipping intermediate details for which the reader is referred to [11], we report now only the conservation properties in the same probabilistic formalism as in (3.6), recalling that kernels fulfill all indistinguishability conditions of the kind: $\Pi_{32}^{2, i}\left(\mathbf{v}^{\prime}, E ; \mathbf{w}^{\prime} \rightarrow \mathbf{v}\right)=\Pi_{23}^{2, i}\left(\mathbf{w}^{\prime} ; \mathbf{v}^{\prime}, E \rightarrow \mathbf{v}\right)$. As regards the inelastic scattering $(I)$ we have

$$
\begin{aligned}
& \int \Pi_{3 p}^{2, i}\left(\mathbf{v}^{\prime}, E ; \mathbf{w}^{\prime} \rightarrow \mathbf{v}\right) d \mathbf{v}=\int \Pi_{p 3}^{p, i}\left(\mathbf{w}^{\prime} ; \mathbf{v}^{\prime}, E \rightarrow \mathbf{v}\right) d \mathbf{v}=1 \\
& \int\left[2 \mathbf{v} \Pi_{3 p}^{2, i}\left(\mathbf{v}^{\prime}, E ; \mathbf{w}^{\prime} \rightarrow \mathbf{v}\right)+p \mathbf{v} \Pi_{p 3}^{p, i}\left(\mathbf{w}^{\prime} ; \mathbf{v}^{\prime}, E \rightarrow \mathbf{v}\right)\right] d \mathbf{v}=2 \mathbf{v}^{\prime}+p \mathbf{w}^{\prime} \\
& \int\left[\left(m_{1} v^{2}-Q\right) \Pi_{3 p}^{2, i}\left(\mathbf{v}^{\prime}, E ; \mathbf{w}^{\prime} \rightarrow \mathbf{v}\right)+\frac{p m_{1}}{2} v^{2} \Pi_{p 3}^{p, i}\left(\mathbf{w}^{\prime} ; \mathbf{v}^{\prime}, E \rightarrow \mathbf{v}\right)\right] d \mathbf{v}= \\
& =m_{1}\left(v^{\prime}\right)^{2}+E+\frac{p m_{1}}{2}\left(w^{\prime}\right)^{2}
\end{aligned}
$$

To the dissociation $(D 1)$ there correspond

$$
\begin{aligned}
& \int \Pi_{2 p}^{1, d}\left(\mathbf{v}^{\prime} ; \mathbf{w}^{\prime} \rightarrow \mathbf{v}\right) d \mathbf{v}=\int \Pi_{p 2}^{p, d}\left(\mathbf{w}^{\prime} ; \mathbf{v}^{\prime} \rightarrow \mathbf{v}\right) d \mathbf{v}=1 \\
& \int\left[2 \mathbf{v} \Pi_{2 p}^{1, d}\left(\mathbf{v}^{\prime} ; \mathbf{w}^{\prime} \rightarrow \mathbf{v}\right)+p \mathbf{v} \Pi_{p 2}^{p, d}\left(\mathbf{w}^{\prime} ; \mathbf{v}^{\prime} \rightarrow \mathbf{v}\right)\right] d \mathbf{v}=2 \mathbf{v}^{\prime}+p \mathbf{w}^{\prime} \\
& \int\left[m_{1} v^{2} \Pi_{2 p}^{1, d}\left(\mathbf{v}^{\prime} ; \mathbf{w}^{\prime} \rightarrow \mathbf{v}\right)+\frac{p m_{1}}{2} v^{2} \Pi_{p 2}^{p, d}\left(\mathbf{w}^{\prime} ; \mathbf{v}^{\prime} \rightarrow \mathbf{v}\right)\right] d \mathbf{v}= \\
& =m_{1}\left(v^{\prime}\right)^{2}-Q+\frac{p m_{1}}{2}\left(w^{\prime}\right)^{2}
\end{aligned}
$$

while for the dissociation $(D 2)$ we have

$$
\begin{aligned}
& \int \Pi_{3 p}^{1, d}\left(\mathbf{v}^{\prime}, E ; \mathbf{w}^{\prime} \rightarrow \mathbf{v}\right) d \mathbf{v}=\int \Pi_{p 3}^{p, d}\left(\mathbf{w}^{\prime} ; \mathbf{v}^{\prime}, E \rightarrow \mathbf{v}\right) d \mathbf{v}=1 \\
& \int\left[2 \mathbf{v} \Pi_{3 p}^{1, d}\left(\mathbf{v}^{\prime}, E ; \mathbf{w}^{\prime} \rightarrow \mathbf{v}\right)+p \mathbf{v} \Pi_{p 3}^{p, d}\left(\mathbf{w}^{\prime} ; \mathbf{v}^{\prime}, E \rightarrow \mathbf{v}\right)\right] d \mathbf{v}=2 \mathbf{v}^{\prime}+p \mathbf{w}^{\prime} \\
& \int\left[m_{1} v^{2} \Pi_{3 p}^{1, d}\left(\mathbf{v}^{\prime}, E ; \mathbf{w}^{\prime} \rightarrow \mathbf{v}\right)+\frac{p m_{1}}{2} v^{2} \Pi_{p 3}^{p, d}\left(\mathbf{w}^{\prime} ; \mathbf{v}^{\prime}, E \rightarrow \mathbf{v}\right)\right] d \mathbf{v}= \\
& =m_{1}\left(v^{\prime}\right)^{2}+E+\frac{p m_{1}}{2}\left(w^{\prime}\right)^{2}
\end{aligned}
$$


Thanks to the conservation properties established in (3.6)-(3.9), if we denote $\mathcal{J}_{1}=$ $\mathcal{J}_{1}^{*}+\mathcal{J}_{1}^{d}+\mathcal{J}_{1}^{r}, \quad \mathcal{J}_{2}=\mathcal{J}_{2}^{*}+\mathcal{J}_{2}^{d}, \mathcal{J}_{3}=\mathcal{J}_{3}^{*}+\mathcal{J}_{3}^{r}$, we get the following conservation laws:

$$
\begin{aligned}
& \int \mathcal{J}_{1}(\mathbf{v}) d \mathbf{v}+2 \int \mathcal{J}_{2}(\mathbf{v}) d \mathbf{v}+2 \iint \mathcal{J}_{3}(\mathbf{v}, E) d E d \mathbf{v}=0 \\
& \int \mathbf{v} \mathcal{J}_{1}(\mathbf{v}) d \mathbf{v}+2 \int \mathbf{v} \mathcal{J}_{2}(\mathbf{v}) d \mathbf{v}+2 \iint \mathbf{v} \mathcal{J}_{3}(\mathbf{v}, E) d E d \mathbf{v}=\mathbf{0} \\
& \int \frac{1}{2} m_{1} v^{2} \mathcal{J}_{1}(\mathbf{v}) d \mathbf{v}+\int\left(m_{1} v^{2}-Q\right) \mathcal{J}_{2}(\mathbf{v}) d \mathbf{v}+\iint\left(m_{1} v^{2}+E\right) \mathcal{J}_{3}(\mathbf{v}, E) d E d \mathbf{v}=0
\end{aligned}
$$

that represent preservation of number of atoms, global momentum, and total (kinetic plus internal) energy, respectively, by chemical reactions not involving the background.

On the other hand, as concerns inelastic scattering or dissociation processes due to encounters with background particles, conservation of atoms remains valid, but we shall have non-vanishing terms in the momentum and energy equations, as typical in linear kinetic theory (see the discussion above about the elastic linear macroscopic contributions). More precisely, the integrations of the relevant scattering kernels yield again

$$
\int \Pi_{30}^{1, d}\left(\mathbf{v}^{\prime}, E ; \mathbf{w}^{\prime} \rightarrow \mathbf{v}\right) d \mathbf{v}=\int \Pi_{20}^{1, d}\left(\mathbf{v}^{\prime} ; \mathbf{w}^{\prime} \rightarrow \mathbf{v}\right) d \mathbf{v}=\int \Pi_{30}^{2, i}\left(\mathbf{v}^{\prime}, E ; \mathbf{w}^{\prime} \rightarrow \mathbf{v}\right) d \mathbf{v}=1
$$

but we cannot have conservation properties as the second and the third in (3.6)-(3.9); we would reobtain the usual conservation laws if the background were not assumed fixed, and its evolution were governed by a kinetic Boltzmann equation. Thus if we denote $\mathcal{I}_{1}=\mathcal{I}_{1}^{*}+\mathcal{I}_{1}^{d}, \quad \mathcal{I}_{2}=\mathcal{I}_{2}^{*}+\mathcal{I}_{2}^{d}, \quad \mathcal{I}_{3}=\mathcal{I}_{3}^{*}$, besides conservation of atoms:

$$
\int \mathcal{I}_{1}(\mathbf{v}) d \mathbf{v}+2 \int \mathcal{I}_{2}(\mathbf{v}) d \mathbf{v}+2 \iint \mathcal{I}_{3}(\mathbf{v}, E) d E d \mathbf{v}=0
$$

some suitable changes of variables allow to cast momentum and energy contributions as

$$
\begin{aligned}
& \int \mathbf{v} \mathcal{I}_{1}(\mathbf{v}) d \mathbf{v}+2 \int \mathbf{v} \mathcal{I}_{2}(\mathbf{v}) d \mathbf{v}+2 \iint \mathbf{v} \mathcal{I}_{3}(\mathbf{v}, E) d E d \mathbf{v}= \\
& =\iiint g_{30}^{d}\left(\left|\mathbf{v}^{\prime}-\mathbf{w}^{\prime}\right|, E\right)\left[\int 2 \mathbf{v} \Pi_{30}^{1, d}\left(\mathbf{v}^{\prime}, E ; \mathbf{w}^{\prime} \rightarrow \mathbf{v}\right) d \mathbf{v}-2 \mathbf{v}^{\prime}\right] \varphi_{3}\left(\mathbf{v}^{\prime}, E\right) f_{0}\left(\mathbf{w}^{\prime}\right) d E d \mathbf{v}^{\prime} d \mathbf{w}^{\prime} \\
& +\iint g_{20}^{d}\left(\left|\mathbf{v}^{\prime}-\mathbf{w}^{\prime}\right|\right)\left[\int 2 \mathbf{v} \Pi_{20}^{1, d}\left(\mathbf{v}^{\prime} ; \mathbf{w}^{\prime} \rightarrow \mathbf{v}\right) d \mathbf{v}-2 \mathbf{v}^{\prime}\right] f_{2}\left(\mathbf{v}^{\prime}\right) f_{0}\left(\mathbf{w}^{\prime}\right) d \mathbf{v}^{\prime} d \mathbf{w}^{\prime} \\
& +\iiint g_{30}^{i}\left(\left|\mathbf{v}^{\prime}-\mathbf{w}^{\prime}\right|, E\right)\left[\int 2 \mathbf{v} \Pi_{30}^{2, i}\left(\mathbf{v}^{\prime}, E ; \mathbf{w}^{\prime} \rightarrow \mathbf{v}\right) d \mathbf{v}-2 \mathbf{v}^{\prime}\right] \varphi_{3}\left(\mathbf{v}^{\prime}, E\right) f_{0}\left(\mathbf{w}^{\prime}\right) d E d \mathbf{v}^{\prime} d \mathbf{w}^{\prime},
\end{aligned}
$$




$$
\begin{aligned}
& \int \frac{1}{2} m_{1} v^{2} \mathcal{I}_{1}(\mathbf{v}) d \mathbf{v}+\int\left(m_{1} v^{2}-Q\right) \mathcal{I}_{2}(\mathbf{v}) d \mathbf{v}+\iint\left(m_{1} v^{2}+E\right) \mathcal{I}_{3}(\mathbf{v}, E) d E d \mathbf{v}= \\
& =\iiint g_{30}^{d}\left(\left|\mathbf{v}^{\prime}-\mathbf{w}^{\prime}\right|, E\right)\left[\int m_{1} v^{2} \Pi_{30}^{1, d}\left(\mathbf{v}^{\prime}, E ; \mathbf{w}^{\prime} \rightarrow \mathbf{v}\right) d \mathbf{v}\right. \\
& \left.-\left(m_{1}\left(v^{\prime}\right)^{2}+E\right)\right] \varphi_{3}\left(\mathbf{v}^{\prime}, E\right) f_{0}\left(\mathbf{w}^{\prime}\right) d E d \mathbf{v}^{\prime} d \mathbf{w}^{\prime} \\
& +\iint g_{20}^{d}\left(\left|\mathbf{v}^{\prime}-\mathbf{w}^{\prime}\right|\right)\left[\int m_{1} v^{2} \Pi_{20}^{1, d}\left(\mathbf{v}^{\prime} ; \mathbf{w}^{\prime} \rightarrow \mathbf{v}\right) d \mathbf{v}-\left(m_{1}\left(v^{\prime}\right)^{2}-Q\right)\right] f_{2}\left(\mathbf{v}^{\prime}\right) f_{0}\left(\mathbf{w}^{\prime}\right) d \mathbf{v}^{\prime} d \mathbf{w}^{\prime} \\
& +\iiint g_{30}^{i}\left(\left|\mathbf{v}^{\prime}-\mathbf{w}^{\prime}\right|, E\right)\left[\int\left(m_{1} v^{2}-Q\right) \Pi_{30}^{2, i}\left(\mathbf{v}^{\prime}, E ; \mathbf{w}^{\prime} \rightarrow \mathbf{v}\right) d \mathbf{v}\right. \\
& \left.-\left(m_{1}\left(v^{\prime}\right)^{2}+E\right)\right] \varphi_{3}\left(\mathbf{v}^{\prime}, E\right) f_{0}\left(\mathbf{w}^{\prime}\right) d E d \mathbf{v}^{\prime} d \mathbf{w}^{\prime} .
\end{aligned}
$$

We can draw immediate consequences at the macroscopic level. At first, integration and summation of kinetic equations (2.1) after multiplication by the string $\left(m_{1}, m_{2}, m_{3}\right)$ yields the conservation of mass density defined as

$$
\rho=m_{1}\left[n_{1}+2\left(n_{2}+n_{3}\right)\right]
$$

with $n_{k}$ standing for the number density of the $k$-species:

$$
n_{k}=\int f_{k}(\mathbf{v}) d \mathbf{v}
$$

Precisely, the continuity equation reads as

$$
\frac{\partial \rho}{\partial t}+\nabla \cdot(\rho \mathbf{u})=0
$$

where $\mathbf{u}$ is the global drift velocity

$$
\mathbf{u}=\frac{1}{\rho} m_{1}\left[n_{1} \mathbf{u}_{1}+2\left(n_{2} \mathbf{u}_{2}+n_{3} \mathbf{u}_{3}\right)\right] \quad \text { with } \quad \mathbf{u}_{k}=\frac{1}{n_{k}} \int \mathbf{v} f_{k}(\mathbf{v}) d \mathbf{v}
$$

Analogously, multiplying the Boltzmann equations by the string $\left(m_{1} \mathbf{v}, m_{2} \mathbf{v}, m_{3} \mathbf{v}\right)$ we get the momentum equation, which is affected by collision contributions (3.2) and 
(3.13) due to (elastic and reactive) scattering with the host medium:

$$
\begin{aligned}
& \frac{\partial}{\partial t}(\rho \mathbf{u})+\nabla \cdot(\rho \mathbf{u} \otimes \mathbf{u}+\mathbf{P})= \\
= & -\frac{2 m_{1} m_{0}}{m_{1}+m_{0}} \iiint b_{10}(\mathbf{v}-\mathbf{w}, \hat{\mathbf{n}})[(\mathbf{v}-\mathbf{w}) \cdot \hat{\mathbf{n}}] \hat{\mathbf{n}} f_{1}(\mathbf{v}) f_{0}(\mathbf{w}) d \mathbf{v} d \mathbf{w} d \hat{\mathbf{n}} \\
& -\frac{4 m_{1} m_{0}}{2 m_{1}+m_{0}} \iiint b_{20}(\mathbf{v}-\mathbf{w}, \hat{\mathbf{n}})[(\mathbf{v}-\mathbf{w}) \cdot \hat{\mathbf{n}}] \hat{\mathbf{n}} f_{2}(\mathbf{v}) f_{0}(\mathbf{w}) d \mathbf{v} d \mathbf{w} d \hat{\mathbf{n}} \\
& +2 m_{1} \iiint g_{30}^{d}\left(\left|\mathbf{v}^{\prime}-\mathbf{w}^{\prime}\right|, E\right)\left[\int \mathbf{v} \Pi_{30}^{1, d}\left(\mathbf{v}^{\prime}, E ; \mathbf{w}^{\prime} \rightarrow \mathbf{v}\right) d \mathbf{v}-\mathbf{v}^{\prime}\right] \varphi_{3}\left(\mathbf{v}^{\prime}, E\right) f_{0}\left(\mathbf{w}^{\prime}\right) d E d \mathbf{v}^{\prime} d \mathbf{w}^{\prime} \\
& +2 m_{1} \iint g_{20}^{d}\left(\left|\mathbf{v}^{\prime}-\mathbf{w}^{\prime}\right|\right)\left[\int \mathbf{v} \Pi_{20}^{1, d}\left(\mathbf{v}^{\prime} ; \mathbf{w}^{\prime} \rightarrow \mathbf{v}\right) d \mathbf{v}-\mathbf{v}^{\prime}\right] f_{2}\left(\mathbf{v}^{\prime}\right) f_{0}\left(\mathbf{w}^{\prime}\right) d \mathbf{v}^{\prime} d \mathbf{w}^{\prime} \\
& +2 m_{1} \iiint g_{30}^{i}\left(\left|\mathbf{v}^{\prime}-\mathbf{w}^{\prime}\right|, E\right)\left[\int \mathbf{v} \Pi_{30}^{2, i}\left(\mathbf{v}^{\prime}, E ; \mathbf{w}^{\prime} \rightarrow \mathbf{v}\right) d \mathbf{v}-\mathbf{v}^{\prime}\right] \varphi_{3}\left(\mathbf{v}^{\prime}, E\right) f_{0}\left(\mathbf{w}^{\prime}\right) d E d \mathbf{v}^{\prime} d \mathbf{w}^{\prime},
\end{aligned}
$$

where $\mathbf{P}$ is the pressure tensor:

$$
\mathbf{P}=\sum_{k=1}^{3} m_{k} \int(\mathbf{v}-\mathbf{u}) \otimes(\mathbf{v}-\mathbf{u}) f_{k}(\mathbf{v}) d \mathbf{v}
$$

Finally, the string $\left(\frac{1}{2} m_{1} v^{2}, \frac{1}{2} m_{2} v^{2}-Q, \frac{1}{2} m_{3} v^{2}+E\right)$ yields the evolution equation for the total internal energy $\mathcal{E}=\frac{1}{2} \rho u^{2}+\mathcal{E}_{\text {th }}+\mathcal{E}_{\text {exc }}$, where thermal and excitation part are given by

$$
\begin{aligned}
& \mathcal{E}_{\mathrm{th}}=\frac{3}{2} n K T=\frac{1}{2} \operatorname{tr}(\mathbf{P}) \quad n=\sum_{k=1}^{3} n_{k} \\
& \mathcal{E}_{\mathrm{exc}}=\int\left[\int E \varphi_{3}(\mathbf{v}, E) d E-Q f_{2}(\mathbf{v})\right] d \mathbf{v} .
\end{aligned}
$$

The streaming terms of the energy equation involve also the heat flux vector $\mathbf{q}=$ $\mathbf{q}_{\mathrm{th}}+\mathbf{q}_{\mathrm{exc}}$, where

$$
\begin{aligned}
& \mathbf{q}_{\mathrm{th}}=\frac{1}{2} \sum_{k=1}^{3} m_{k} \int(\mathbf{v}-\mathbf{u})^{2}(\mathbf{v}-\mathbf{u}) f_{k}(\mathbf{v}) d \mathbf{v}, \\
& \mathbf{q}_{\mathrm{exc}}=\int(\mathbf{v}-\mathbf{u})\left[\int E \varphi_{3}(\mathbf{v}, E) d E-Q f_{2}(\mathbf{v})\right] d \mathbf{v},
\end{aligned}
$$

while collision contributions are essentially provided by (3.3) and (3.14):

$$
\begin{aligned}
& \frac{\partial \mathcal{E}}{\partial t}+\nabla \cdot[\mathcal{E} \mathbf{u}+\mathbf{P} \cdot \mathbf{u}+\mathbf{q}]= \\
&=\frac{2 m_{1} m_{0}}{m_{1}+m_{0}} \iiint b_{10}(\mathbf{v}-\mathbf{w}, \hat{\mathbf{n}})\left\{\frac{m_{0}}{m_{1}+m_{0}}[(\mathbf{v}-\mathbf{w}) \cdot \hat{\mathbf{n}}]^{2}\right. \\
&-[(\mathbf{v}-\mathbf{w}) \cdot \hat{\mathbf{n}}](\mathbf{v} \cdot \hat{\mathbf{n}})\} f_{1}(\mathbf{v}) f_{0}(\mathbf{w}) d \mathbf{v} d \mathbf{w} d \hat{\mathbf{n}}
\end{aligned}
$$




$$
\begin{aligned}
& +\frac{4 m_{1} m_{0}}{2 m_{1}+m_{0}} \iiint b_{20}(\mathbf{v}-\mathbf{w}, \hat{\mathbf{n}})\left\{\frac{m_{0}}{2 m_{1}+m_{0}}[(\mathbf{v}-\mathbf{w}) \cdot \hat{\mathbf{n}}]^{2}\right. \\
& -[(\mathbf{v}-\mathbf{w}) \cdot \hat{\mathbf{n}}](\mathbf{v} \cdot \hat{\mathbf{n}})\} f_{2}(\mathbf{v}) f_{0}(\mathbf{w}) d \mathbf{v} d \mathbf{w} d \hat{\mathbf{n}} \\
& +\iiint g_{30}^{d}\left(\left|\mathbf{v}^{\prime}-\mathbf{w}^{\prime}\right|, E\right)\left[\int m_{1} v^{2} \Pi_{30}^{1, d}\left(\mathbf{v}^{\prime}, E ; \mathbf{w}^{\prime} \rightarrow \mathbf{v}\right) d \mathbf{v}\right. \\
& \left.\quad-\left(m_{1}\left(v^{\prime}\right)^{2}+E\right)\right] \varphi_{3}\left(\mathbf{v}^{\prime}, E\right) f_{0}\left(\mathbf{w}^{\prime}\right) d E d \mathbf{v}^{\prime} d \mathbf{w}^{\prime} \\
& +\iint g_{20}^{d}\left(\left|\mathbf{v}^{\prime}-\mathbf{w}^{\prime}\right|\right)\left[\int m_{1} v^{2} \Pi_{20}^{1, d}\left(\mathbf{v}^{\prime} ; \mathbf{w}^{\prime} \rightarrow \mathbf{v}\right) d \mathbf{v}-\left(m_{1}\left(v^{\prime}\right)^{2}-Q\right)\right] \\
& \quad \cdot f_{2}\left(\mathbf{v}^{\prime}\right) f_{0}\left(\mathbf{w}^{\prime}\right) d \mathbf{v}^{\prime} d \mathbf{w}^{\prime} \\
& +\iiint g_{30}^{i}\left(\left|\mathbf{v}^{\prime}-\mathbf{w}^{\prime}\right|, E\right)\left[\int\left(m_{1} v^{2}-Q\right) \Pi_{30}^{2, i}\left(\mathbf{v}^{\prime}, E ; \mathbf{w}^{\prime} \rightarrow \mathbf{v}\right) d \mathbf{v}\right. \\
& \left.\quad-\left(m_{1}\left(v^{\prime}\right)^{2}+E\right)\right] \varphi_{3}\left(\mathbf{v}^{\prime}, E\right) f_{0}\left(\mathbf{w}^{\prime}\right) d E d \mathbf{v}^{\prime} d \mathbf{w}^{\prime} .
\end{aligned}
$$

The five scalar partial differential equations (3.15), (3.16), (3.17) are of course exact but not closed, since they involve a larger number of unknown fields (moments of the distribution functions), and moreover the collision contributions appearing in the momentum and in the energy equations are in general not directly amenable to macroscopic quantities.

From now on we shall assume that the intermolecular forces are of Maxwell molecules type [4], namely proportional to the power $d^{-5}$, where $d$ is the intermolecular distance. This choice implies that the differential collision frequencies (both elastic and chemical) depend only on the angle formed by $\hat{\mathbf{n}}$ and the relative velocity, and consequently total collision frequencies turn out to be constant, and will be denoted by the symbol $\nu_{i j}^{k}$. Under these assumptions, momentum and energy balance equations can be rewritten in a simpler form. As concerns elastic operators, the total collision frequencies are given by

$$
\nu_{j 0}=\int b_{j 0}\left(\frac{\mathbf{v}-\mathbf{w}}{|\mathbf{v}-\mathbf{w}|} \cdot \hat{\mathbf{n}}\right) d \hat{\mathbf{n}},
$$

and integrations over the angular variable $\hat{\mathbf{n}}$ in elastic contributions (3.2) and (3.3) can be explicitly cast in terms of the suitably weighted collision frequency

$$
\bar{\nu}_{j 0}=2 \pi \int_{0}^{\pi / 2} b_{j 0}(\theta) \cos ^{2} \theta \sin \theta d \theta<\nu_{j 0},
$$

where $\theta$ represents the impact angle between $\mathbf{v}-\mathbf{w}$ and $\hat{\mathbf{n}}$. Precisely, the required integrals are amenable to

$$
\begin{aligned}
& \int b_{j 0}\left(\frac{\mathbf{v}-\mathbf{w}}{|\mathbf{v}-\mathbf{w}|} \cdot \hat{\mathbf{n}}\right)[(\mathbf{v}-\mathbf{w}) \cdot \hat{\mathbf{n}}] \hat{\mathbf{n}} d \hat{\mathbf{n}}=\bar{\nu}_{j 0}(\mathbf{v}-\mathbf{w}), \\
& \int b_{j 0}\left(\frac{\mathbf{v}-\mathbf{w}}{|\mathbf{v}-\mathbf{w}|} \cdot \hat{\mathbf{n}}\right)[(\mathbf{v}-\mathbf{w}) \cdot \hat{\mathbf{n}}]^{2} d \hat{\mathbf{n}}=\bar{\nu}_{j 0}(\mathbf{v}-\mathbf{w})^{2}
\end{aligned}
$$


In conclusion, the momentum equation for Maxwellian molecules is

$$
\begin{aligned}
& \quad \frac{\partial}{\partial t}(\rho \mathbf{u})+\nabla \cdot(\rho \mathbf{u} \otimes \mathbf{u}+\mathbf{P})= \\
& =-\frac{2 m_{1} m_{0}}{m_{1}+m_{0}} \bar{\nu}_{10} \iint(\mathbf{v}-\mathbf{w}) f_{1}(\mathbf{v}) f_{0}(\mathbf{w}) d \mathbf{v} d \mathbf{w} \\
& \quad-\frac{4 m_{1} m_{0}}{2 m_{1}+m_{0}} \bar{\nu}_{20} \iint(\mathbf{v}-\mathbf{w}) f_{2}(\mathbf{v}) f_{0}(\mathbf{w}) d \mathbf{v} d \mathbf{w} \\
& \quad+2 m_{1} \nu_{30}^{d} \iiint\left[\int \mathbf{v} \Pi_{30}^{1, d}\left(\mathbf{v}^{\prime}, E ; \mathbf{w}^{\prime} \rightarrow \mathbf{v}\right) d \mathbf{v}-\mathbf{v}^{\prime}\right] \varphi_{3}\left(\mathbf{v}^{\prime}, E\right) f_{0}\left(\mathbf{w}^{\prime}\right) d E d \mathbf{v}^{\prime} d \mathbf{w}^{\prime} \\
& \quad+2 m_{1} \nu_{20}^{d} \iint\left[\int \mathbf{v} \Pi_{20}^{1, d}\left(\mathbf{v}^{\prime} ; \mathbf{w}^{\prime} \rightarrow \mathbf{v}\right) d \mathbf{v}-\mathbf{v}^{\prime}\right] f_{2}\left(\mathbf{v}^{\prime}\right) f_{0}\left(\mathbf{w}^{\prime}\right) d \mathbf{v}^{\prime} d \mathbf{w}^{\prime} \\
& \quad+2 m_{1} \nu_{30}^{i} \iiint\left[\int \mathbf{v} \Pi_{30}^{2, i}\left(\mathbf{v}^{\prime}, E ; \mathbf{w}^{\prime} \rightarrow \mathbf{v}\right) d \mathbf{v}-\mathbf{v}^{\prime}\right] \varphi_{3}\left(\mathbf{v}^{\prime}, E\right) f_{0}\left(\mathbf{w}^{\prime}\right) d E d \mathbf{v}^{\prime} d \mathbf{w}^{\prime},
\end{aligned}
$$

while the energy equation takes the form

$$
\begin{aligned}
& \frac{\partial \mathcal{E}}{\partial t}+\nabla \cdot[\mathcal{E} \mathbf{u}+\mathbf{P} \cdot \mathbf{u}+\mathbf{q}]= \\
& =\frac{2 m_{1} m_{0}}{m_{1}+m_{0}} \bar{\nu}_{10} \iint\left\{\frac{m_{0}}{m_{1}+m_{0}}(\mathbf{v}-\mathbf{w})^{2}-(\mathbf{v}-\mathbf{w}) \cdot \mathbf{v}\right\} f_{1}(\mathbf{v}) f_{0}(\mathbf{w}) d \mathbf{v} d \mathbf{w} \\
& \quad+\frac{4 m_{1} m_{0}}{2 m_{1}+m_{0}} \bar{\nu}_{20} \iint\left\{\frac{m_{0}}{2 m_{1}+m_{0}}(\mathbf{v}-\mathbf{w})^{2}-(\mathbf{v}-\mathbf{w}) \cdot \mathbf{v}\right\} f_{2}(\mathbf{v}) f_{0}(\mathbf{w}) d \mathbf{v} d \mathbf{w} \\
& \quad+\nu_{30}^{d} \iiint\left[\int m_{1} v^{2} \Pi_{30}^{1, d}\left(\mathbf{v}^{\prime}, E ; \mathbf{w}^{\prime} \rightarrow \mathbf{v}\right) d \mathbf{v}\right. \\
& \left.\quad-\left(m_{1}\left(v^{\prime}\right)^{2}+E\right)\right] \varphi_{3}\left(\mathbf{v}^{\prime}, E\right) f_{0}\left(\mathbf{w}^{\prime}\right) d E d \mathbf{v}^{\prime} d \mathbf{w}^{\prime} \\
& \quad+\nu_{20}^{d} \iint\left[\int m_{1} v^{2} \Pi_{20}^{1, d}\left(\mathbf{v}^{\prime} ; \mathbf{w}^{\prime} \rightarrow \mathbf{v}\right) d \mathbf{v}-\left(m_{1}\left(v^{\prime}\right)^{2}-Q\right)\right] f_{2}\left(\mathbf{v}^{\prime}\right) f_{0}\left(\mathbf{w}^{\prime}\right) d \mathbf{v}^{\prime} d \mathbf{w}^{\prime} \\
& \quad-\left(\nu _ { 3 0 } ^ { i } \int \int \int \left[\int\left(m_{1} v^{2}-Q\right) \Pi_{30}^{2, i}\left(\mathbf{v}^{\prime}, E ; \mathbf{w}^{\prime} \rightarrow \mathbf{v}\right) d \mathbf{v}\right.\right. \\
& \quad-E)] \varphi_{3}\left(\mathbf{v}^{\prime}, E\right) f_{0}\left(\mathbf{w}^{\prime}\right) d E d \mathbf{v}^{\prime} d \mathbf{w}^{\prime} .
\end{aligned}
$$

Notice that the elastic contributions and the "loss part" of the chemical terms (not involving the scattering kernels $\Pi_{i j}^{h, \mathrm{k}}$ ) might be expressed, wherever convenient, in terms of main macroscopic moments of single interacting species (number densities $n_{k}$, mean velocities $\mathbf{u}_{k}$ and temperatures $T_{k}$ ).

\section{Reaction-diffusion asymptotic limit}

As usual in kinetic theory, to build up consistent hydrodynamic equations for the main macroscopic fields we shall analyze the kinetic Boltzmann equations (2.1) in dimensionless form. At the kinetic level, the main effect of the adimensionalization is the appearance of some ratios involving the different scales that measure the relative 
importance of each operator during the evolution of the mixture. Such ratios can be expressed in terms of a small parameter $\epsilon$, that often is one of them, typically the Knudsen number. In the equations for atoms $A$ and for stable molecules $A_{2}$, we shall assume that the elastic scattering with the background medium plays a crucial role, so that the linear elastic operators shall be taken of order $O(1 / \epsilon)$. This choice, introduced and justified in many papers [1], is quite reasonable from a physical point of view, since the host medium is much denser than the other species. Then, each elastic operator $Q_{i j}$ with $i, j \neq 0$ shall be $O(1)$ (slower than linear ones). Reactive processes are usually assumed less frequent than elastic scattering, since reactions involve more complicated microscopic dynamics such as change of chemical nature (mass transfer) and mutual exchange of internal energy. However, chemical operators $\mathcal{J}_{i}^{*}$ and $\mathcal{I}_{i}^{*}(i=1,2)$ involving the unstable molecules distribution will be supposed significantly faster than other chemical processes, because of the very small mean free path typical of the transition state $A_{2}^{*}$, so that it disappears nearly immediately after creation. This is indeed consistent with the present physical model, in which the process is characterized and driven by a transition state $[11,18]$. So, dissociation and recombination operators $\mathcal{J}_{i}^{r}, \mathcal{J}_{i}^{d}$ and $\mathcal{I}_{i}^{d}$ will be taken of order $\epsilon$, as usual in order to recover the time-scale of diffusion $[7,17,19,1]$, while $\mathcal{J}_{i}^{*}$ and $\mathcal{I}_{i}^{*}$ (depending on the distribution $\varphi_{3}$ ) will be affected by a factor $1 / \epsilon$. This means that decay times of the unstable molecules is $O\left(\epsilon^{2}\right)$ with respect to the other chemical relaxation times. This also means that chemical collision frequencies with the background are smaller by a factor $\epsilon$ than the corresponding collision frequencies of the participating species between themselves. In this regime, the dominant role in the evolution of atoms and of stable molecules is played both by elastic collisions with the host medium, and by chemical reactions involving an unstable molecule among the ingoing particles. Consequently, in the kinetic equation for the distribution $\varphi_{3}$, the chemical terms involving $\varphi_{3}$ itself are assumed $O(1 / \epsilon)$, while the remaining scattering operator $\mathcal{J}_{3}^{r}$ remains $O(\epsilon)$. Finally, since we are interested in the time scale of chemical reactions, including the slower ones, we put the scaling $\epsilon$ in front of the temporal derivatives [1]. Actually the present scaling can be explicitly achieved by adimensionalization upon introducing typical or average values for species densities $\bar{n}$, background density $\bar{n}_{0}$, scattering cross sections $\bar{\sigma}_{S}$ and $\bar{\sigma}_{0 S}$ (for species and background), reacting cross sections $\bar{\sigma}_{C}$ and $\bar{\sigma}_{0 C}$ (not involving the unstable species), and $\bar{\sigma}_{C}^{*}$ and $\bar{\sigma}_{0 C}^{*}$ (involving the transition state). Now, if $\xi$ denotes the scale of molecular velocities, and we take $\bar{\sigma}_{0 S}=\bar{\sigma}_{S}$, we can use the Knudsen number $K n=\bar{n} / \bar{n}_{0}$ as small parameter $\epsilon\left(\bar{n}_{0}>>\bar{n}\right)$, measuring lengths in units of $\left(\bar{n} \bar{\sigma}_{S}\right)^{-1}$, times in units of $\left(\xi \bar{n}_{0} \bar{\sigma}_{S}\right)^{-1}$, and bearing in mind that our physical problem is characterized by $\bar{\sigma}_{C}^{*} / \bar{\sigma}_{S}=O(1 / \epsilon), \bar{\sigma}_{C} / \bar{\sigma}_{S}=O(\epsilon)$, $\bar{\sigma}_{0 C}^{*} / \bar{\sigma}_{S}=O(1), \bar{\sigma}_{0 C} / \bar{\sigma}_{S}=O\left(\epsilon^{2}\right)$. Of course analogous scaling could be devised for other physical conditions, with results that would be either similar to or different from the present ones. The question will be matter of future work.

In any case, under our assumptions, the re-scaled kinetic system reads as

$$
\begin{aligned}
\epsilon \frac{\partial f_{1}}{\partial t}+\mathbf{v} \cdot \frac{\partial f_{1}}{\partial \mathbf{x}}= & \frac{1}{\epsilon} \mathcal{Q}_{10}\left(f_{1}, f_{0}\right)+\mathcal{Q}_{11}\left(f_{1}\right)+\mathcal{Q}_{12}\left(f_{1}, f_{2}\right) \\
& +\frac{1}{\epsilon} \mathcal{J}_{1}^{*}\left(f_{1}, f_{2}, \varphi_{3}\right)+\epsilon \mathcal{J}_{1}^{d}\left(f_{1}, f_{2}\right)+\epsilon \mathcal{J}_{1}^{r}\left(f_{1}\right)+\frac{1}{\epsilon} \mathcal{I}_{1}^{*}\left(\varphi_{3}, f_{0}\right)+\epsilon \mathcal{I}_{1}^{d}\left(f_{2}, f_{0}\right), \\
\epsilon \frac{\partial f_{2}}{\partial t}+\mathbf{v} \cdot \frac{\partial f_{2}}{\partial \mathbf{x}}= & \frac{1}{\epsilon} \mathcal{Q}_{20}\left(f_{2}, f_{0}\right)+\mathcal{Q}_{21}\left(f_{2}, f_{1}\right)+\mathcal{Q}_{22}\left(f_{2}\right)
\end{aligned}
$$




$$
\begin{aligned}
& +\frac{1}{\epsilon} \mathcal{J}_{2}^{*}\left(f_{1}, f_{2}, \varphi_{3}\right)+\epsilon \mathcal{J}_{2}^{d}\left(f_{1}, f_{2}\right)+\frac{1}{\epsilon} \mathcal{I}_{2}^{*}\left(\varphi_{3}, f_{0}\right)+\epsilon \mathcal{I}_{2}^{d}\left(f_{2}, f_{0}\right), \\
\epsilon \frac{\partial \varphi_{3}}{\partial t}+\mathbf{v} \cdot \frac{\partial \varphi_{3}}{\partial \mathbf{x}}= & \frac{1}{\epsilon} \mathcal{J}_{3}^{*}\left(f_{1}, f_{2}, \varphi_{3}\right)+\epsilon \mathcal{J}_{3}^{r}\left(f_{1}\right)+\frac{1}{\epsilon} \mathcal{I}_{3}^{*}\left(\varphi_{3}, f_{0}\right) .
\end{aligned}
$$

Let us perform an asymptotic analysis of these equations, in order to build up a consistent set of closed evolution equations for the macroscopic fields $n_{1}, n_{2}$. We deal here with Maxwellian molecules, for which coefficients appearing in the final macroscopic equations may be evaluated in an explicit form. However, the main steps of the procedure outlined in this section could be repeated, with of course some additional technical difficulties, also for general cross sections [1]. Moreover, since the background medium is assumed fixed (stationary and homogeneous), we may set without loss of generality $\mathbf{u}_{0}=\mathbf{0}$. At first, it is worth analyzing the third equation in (4.1); the chemical operators $\mathcal{J}_{3}^{*}$ and $\mathcal{I}_{3}^{*}$ are the dominant ones, in the sense that

$$
\mathcal{J}_{3}^{*}\left(f_{1}, f_{2}, \varphi_{3}\right)+\mathcal{I}_{3}^{*}\left(\varphi_{3}, f_{0}\right)=O(\epsilon) .
$$

Since both operators are constituted by the function $\varphi_{3}(\mathbf{v}, E)$ multiplied by negative factors (see (2.9) and (2.15)) and in $\mathcal{I}_{3}^{*}$ such factor is actually $O(1)$, we get that to the leading order the distribution $\varphi_{3}$ vanishes, so we have $\varphi_{3}=\epsilon \varphi_{3}^{(1)}$, consistently with the fact that unstable molecules easily disappear. This result has several consequences going on in the asymptotic procedure. First of all, in the equations for $f_{1}$ and $f_{2}$ the linear elastic scattering is actually the dominant process:

$$
\mathcal{Q}_{10}\left(f_{1}, n_{0} M_{0}\right)=O(\epsilon), \quad \mathcal{Q}_{20}\left(f_{2}, n_{0} M_{0}\right)=O(\epsilon),
$$

where $M_{0}$ is the background Maxwellian distribution (1.1) (with $\mathbf{u}_{0}=\mathbf{0}$ ). As known in the literature, the linear operator $L_{i}=\mathcal{Q}_{i 0}\left(\cdot, M_{0}\right)$ is bounded, self-adjoint and Fredholm in $L^{2}\left(M^{-1}(\mathbf{v}) d \mathbf{v}\right)$ (this is a consequence of the computations in [4] for example). Moreover, according to the linear $H$-theorem, the spectrum of $L_{i}$ is included in $\mathbb{R}^{-}$, and 0 is an eigenvalue of order 1 whose eigenfunction is the Maxwellian function

$$
M_{i}=\left(\frac{m_{i}}{2 \pi T_{0}}\right)^{\frac{3}{2}} \exp \left(-\frac{m_{i}}{2 T_{0}}|\mathbf{v}|^{2}\right),
$$

having the same zero mean velocity and temperature $T_{0}$ as the fixed background. As a consequence, $f_{1}$ and $f_{2}$ are a perturbation of order 1 of a collision equilibrium:

$$
\begin{aligned}
& f_{1}(t, \mathbf{x}, \mathbf{v})=n_{1}(t, \mathbf{x}) M_{1}(\mathbf{v})+\epsilon f_{1}^{(1)}(t, \mathbf{x}, \mathbf{v}), \\
& f_{2}(t, \mathbf{x}, \mathbf{v})=n_{2}(t, \mathbf{x}) M_{2}(\mathbf{v})+\epsilon f_{2}^{(1)}(t, \mathbf{x}, \mathbf{v}),
\end{aligned}
$$

where without loss of generality we may assume that the actual number densities $n_{1}, n_{2}$ affect the leading order Maxwellian, while the $O(\epsilon)$ corrections have vanishing density:

$$
\int f_{1}^{(1)}(\mathbf{v}) d \mathbf{v}=\int f_{2}^{(1)}(\mathbf{v}) d \mathbf{v}=0 .
$$

Moreover, the result $\varphi_{3}=\epsilon \varphi_{3}^{(1)}$ implies that in the third equation (4.1) leading order streaming terms vanish, so that next step of the asymptotic procedure reads analogously to (4.2), only with $\varphi_{3}^{(1)}$ in place of $\varphi_{3}$, thus it yields $\varphi_{3}^{(1)}=\epsilon \varphi_{3}^{(2)}$ namely

$$
\varphi_{3}(t, \mathbf{x}, \mathbf{v}, E)=\epsilon^{2} \varphi_{3}^{(2)}(t, \mathbf{x}, \mathbf{v}, E) .
$$


At this point, the third of (4.1) reads as

$$
\mathcal{J}_{3}^{*}\left(f_{1}, f_{2}, \varphi_{3}^{(2)}\right)+\mathcal{J}_{3}^{r}\left(f_{1}\right)+\mathcal{I}_{3}^{*}\left(\varphi_{3}^{(2)}, f_{0}\right)=O(\epsilon),
$$

from which, taking into account (4.5), we can derive an explicit expression for (the leading order of) the perturbation $\varphi_{3}^{(2)}$ :

$$
\begin{gathered}
\varphi_{3}^{(2)}(\mathbf{v}, E)=\left[\left(n_{1}\right)^{2} \iint g_{11}^{r}\left(\left|\mathbf{v}^{\prime}-\mathbf{w}^{\prime}\right|\right) \Pi_{11}^{3, r}\left(\mathbf{v}^{\prime} ; \mathbf{w}^{\prime} \rightarrow \mathbf{v}, E\right) M_{1}\left(\mathbf{v}^{\prime}\right) M_{1}\left(\mathbf{w}^{\prime}\right) d \mathbf{v}^{\prime} d \mathbf{w}^{\prime}\right] \\
\cdot\left[n_{1} \int g_{31}^{t}(|\mathbf{v}-\mathbf{w}|, E) M_{1}(\mathbf{w}) d \mathbf{w}+n_{2} \int g_{32}^{t}(|\mathbf{v}-\mathbf{w}|, E) M_{2}(\mathbf{w}) d \mathbf{w}\right. \\
\left.+n_{0} \int g_{30}^{t}(|\mathbf{v}-\mathbf{w}|, E) M_{0}(\mathbf{w}) d \mathbf{w}\right]^{-1}+O(\epsilon)
\end{gathered}
$$

where

$$
g_{3 j}^{t}=g_{3 j}^{d}+g_{3 j}^{i} .
$$

Recalling that in the Maxwell molecules case the total collision frequencies $g_{i j}^{k}$ are constants (denoted by $\nu_{i j}^{\mathrm{k}}$ ), the function $\varphi_{3}^{(2)}$ can be given in a simpler fashion:

$\varphi_{3}^{(2)}(\mathbf{v}, E)=\frac{\nu_{11}^{r}\left(n_{1}\right)^{2}}{\nu_{30}^{t} n_{0}+\nu_{31}^{t} n_{1}+\nu_{32}^{t} n_{2}} \iint \Pi_{11}^{3, r}\left(\mathbf{v}^{\prime} ; \mathbf{w}^{\prime} \rightarrow \mathbf{v}, E\right) M_{1}\left(\mathbf{v}^{\prime}\right) M_{1}\left(\mathbf{w}^{\prime}\right) d \mathbf{v}^{\prime} d \mathbf{w}^{\prime}+O(\epsilon)$.

Moreover, bearing in mind that the scattering kernel $\Pi_{11}^{3, r}$ has the explicit form (3.5), the integration in the velocity variables can be solved, and in conclusion we have

$$
\varphi_{3}^{(2)}(\mathbf{v}, E)=\frac{\nu_{11}^{r}\left(n_{1}\right)^{2}}{\nu_{30}^{t} n_{0}+\nu_{31}^{t} n_{1}+\nu_{32}^{t} n_{2}} M_{3}(\mathbf{v}) \chi(E)+O(\epsilon),
$$

where $M_{3}(\mathbf{v})$ is the normalized Maxwellian (4.4) and the energy spectrum

$$
\chi(E)=2\left(\frac{E}{\pi\left(T_{0}\right)^{3}}\right)^{\frac{1}{2}} \exp \left(-\frac{E}{T_{0}}\right),
$$

satisfies the normalization condition

$$
\int \chi(E) d E=1 .
$$

A consequence of (4.10) is that $n_{3}=\epsilon^{2} n_{3}^{(2)}+O\left(\epsilon^{3}\right)$, with

$$
n_{3}^{(2)}=\iint \varphi_{3}^{(2)}(\mathbf{v}, E) d E d \mathbf{v}=\frac{\nu_{11}^{r}\left(n_{1}\right)^{2}}{\nu_{30}^{t} n_{0}+\nu_{31}^{t} n_{1}+\nu_{32}^{t} n_{2}} .
$$

The balance equations for the number densities $n_{1}, n_{2}$ shall be obtained, as usual, by integrating the kinetic equations for $f_{1}, f_{2}$ in the velocity variable. The evaluation of relevant streaming and scattering contributions, together with the closure procedure to the first order accuracy, will be explained in detail for species 1 (atoms $A$ ). The same steps may then be performed as regards species 2 (molecules $A_{2}$ ), for which we 
shall report only the final results. Integrating the first equation in (4.1) we get the continuity equation

$$
\begin{gathered}
\epsilon \frac{\partial}{\partial t} \int f_{1}(\mathbf{v}) d \mathbf{v}+\frac{\partial}{\partial \mathbf{x}} \cdot \int \mathbf{v} f_{1}(\mathbf{v}) d \mathbf{v}=\frac{1}{\epsilon} \int \mathcal{J}_{1}^{*}\left(f_{1}, f_{2}, \varphi_{3}\right)(\mathbf{v}) d \mathbf{v}+\epsilon \int \mathcal{J}_{1}^{d}\left(f_{1}, f_{2}\right)(\mathbf{v}) d \mathbf{v} \\
+\epsilon \int \mathcal{J}_{1}^{r}\left(f_{1}\right)(\mathbf{v}) d \mathbf{v}+\frac{1}{\epsilon} \int \mathcal{I}_{1}^{*}\left(\varphi_{3}, f_{0}\right)(\mathbf{v}) d \mathbf{v}+\epsilon \int \mathcal{I}_{1}^{d}\left(f_{2}, f_{0}\right)(\mathbf{v}) d \mathbf{v}
\end{gathered}
$$

where elastic contributions disappear thanks to the preservation of particles by elastic collisions, and where, as usual, also the momentum $\int \mathbf{v} f_{1}(\mathbf{v}) d \mathbf{v}$ appears. In order to evaluate it in terms of the number densities and of background fields, in the Maxwell molecule frame it is convenient to resort to the momentum equation, achieved as weak form of the Boltzmann equation corresponding to the weight function $\mathbf{v}$ :

$$
\begin{aligned}
& \epsilon \frac{\partial}{\partial t} \int \mathbf{v} f_{1}(\mathbf{v}) d \mathbf{v}+\frac{\partial}{\partial \mathbf{x}} \cdot \int(\mathbf{v} \otimes \mathbf{v}) f_{1}(\mathbf{v}) d \mathbf{v}= \\
= & \frac{1}{\epsilon} \int \mathbf{v} \mathcal{Q}_{10}\left(f_{1}, f_{0}\right)(\mathbf{v}) d \mathbf{v}+\int \mathbf{v} \mathcal{Q}_{11}\left(f_{1}\right)(\mathbf{v}) d \mathbf{v}+\int \mathbf{v} \mathcal{Q}_{12}\left(f_{1}, f_{2}\right)(\mathbf{v}) d \mathbf{v} \\
& +\frac{1}{\epsilon} \int \mathbf{v} \mathcal{J}_{1}^{*}\left(f_{1}, f_{2}, \varphi_{3}\right)(\mathbf{v}) d \mathbf{v}+\epsilon \int \mathbf{v} \mathcal{J}_{1}^{d}\left(f_{1}, f_{2}\right)(\mathbf{v}) d \mathbf{v}+\epsilon \int \mathbf{v} \mathcal{J}_{1}^{r}\left(f_{1}\right)(\mathbf{v}) d \mathbf{v} \\
& +\frac{1}{\epsilon} \int \mathbf{v} \mathcal{I}_{1}^{*}\left(\varphi_{3}, f_{0}\right)(\mathbf{v}) d \mathbf{v}+\epsilon \int \mathbf{v} \mathcal{I}_{1}^{d}\left(f_{2}, f_{0}\right)(\mathbf{v}) d \mathbf{v} .
\end{aligned}
$$

As shown in the previous section (see equalities (3.2) and (3.18)), the collision contribution due to the dominant operator (the linear elastic one) can be cast, for Maxwellian molecules, as

$$
\begin{aligned}
\int \mathbf{v} \mathcal{Q}_{10}\left(f_{1}, f_{0}\right)(\mathbf{v}) d \mathbf{v} & =-\frac{2 m_{0}}{m_{1}+m_{0}} \bar{\nu}_{10} \iint(\mathbf{v}-\mathbf{w}) f_{1}(\mathbf{v}) f_{0}(\mathbf{w}) d \mathbf{v} d \mathbf{w}= \\
& =-\frac{2 m_{0}}{m_{1}+m_{0}} \bar{\nu}_{10} n_{0} \int \mathbf{v} f_{1}(\mathbf{v}) d \mathbf{v}
\end{aligned}
$$

where the last equality holds since we are assuming vanishing background drift velocity. At this point, notice that last integral in (4.15) is just the momentum needed in the continuity equation (4.13), hence it can be "extracted" from the momentum equation (4.14) and inserted into (4.13), ending up with

$$
\begin{gathered}
\frac{\partial}{\partial t} \int f_{1}(\mathbf{v}) d \mathbf{v}+\frac{m_{1}+m_{0}}{2 m_{0}} \frac{1}{\bar{\nu}_{10} n_{0}} \frac{\partial}{\partial \mathbf{x}} \cdot\left\{-\epsilon \frac{\partial}{\partial t} \int \mathbf{v} f_{1}(\mathbf{v}) d \mathbf{v}-\frac{\partial}{\partial \mathbf{x}} \cdot \int(\mathbf{v} \otimes \mathbf{v}) f_{1}(\mathbf{v}) d \mathbf{v}\right. \\
+\int \mathbf{v} \mathcal{Q}_{11}\left(f_{1}\right)(\mathbf{v}) d \mathbf{v}+\int \mathbf{v} \mathcal{Q}_{12}\left(f_{1}, f_{2}\right)(\mathbf{v}) d \mathbf{v}+\epsilon \int \mathbf{v} \mathcal{J}_{1}^{*}\left(f_{1}, f_{2}, \varphi_{3}^{(2)}\right)(\mathbf{v}) d \mathbf{v} \\
+\epsilon \int \mathbf{v} \mathcal{J}_{1}^{d}\left(f_{1}, f_{2}\right)(\mathbf{v}) d \mathbf{v}+\epsilon \int \mathbf{v} \mathcal{J}_{1}^{r}\left(f_{1}\right)(\mathbf{v}) d \mathbf{v}+\epsilon \int \mathbf{v} \mathcal{I}_{1}^{*}\left(\varphi_{3}^{(2)}, f_{0}\right)(\mathbf{v}) d \mathbf{v} \\
\left.+\epsilon \int \mathbf{v} \mathcal{I}_{1}^{d}\left(f_{2}, f_{0}\right)(\mathbf{v}) d \mathbf{v}\right\}=
\end{gathered}
$$




$$
\begin{aligned}
= & \int \mathcal{J}_{1}^{*}\left(f_{1}, f_{2}, \varphi_{3}^{(2)}\right)(\mathbf{v}) d \mathbf{v}+\int \mathcal{J}_{1}^{d}\left(f_{1}, f_{2}\right)(\mathbf{v}) d \mathbf{v} \\
& +\int \mathcal{J}_{1}^{r}\left(f_{1}\right)(\mathbf{v}) d \mathbf{v}+\int \mathcal{I}_{1}^{*}\left(\varphi_{3}^{(2)}, f_{0}\right)(\mathbf{v}) d \mathbf{v}+\int \mathcal{I}_{1}^{d}\left(f_{2}, f_{0}\right)(\mathbf{v}) d \mathbf{v} .
\end{aligned}
$$

Bearing in mind that $f_{1}, f_{2}$ take the form (4.5), namely they are perturbations of a Maxwellian distribution, the leading order pressure tensor takes the isotropic form

$$
\int(\mathbf{v} \otimes \mathbf{v}) f_{1}(\mathbf{v}) d \mathbf{v}=\frac{1}{m_{1}} n_{1} T_{0} \mathbf{I}+O(\epsilon),
$$

where $\mathbf{I}$ is the identity tensor, while leading order elastic contributions on the left hand side of (4.16) vanish:

$$
\int \mathbf{v} \mathcal{Q}_{11}\left(f_{1}\right)(\mathbf{v}) d \mathbf{v}=O(\epsilon), \quad \quad \int \mathbf{v} \mathcal{Q}_{12}\left(f_{1}, f_{2}\right)(\mathbf{v}) d \mathbf{v}=O(\epsilon),
$$

(the second equality holds since $M_{1}$ and $M_{2}$ share the same drift velocity), thus the macroscopic equation (4.16) does not contain $O(1)$ elastic contributions. Finally, we have to evaluate the $O(1)$ chemical terms appearing on the right hand side. In the Maxwell molecules frame (with constant collision frequencies), taking into account that $f_{1}=n_{1} M_{1}+O(\epsilon), f_{2}=n_{2} M_{2}+O(\epsilon)$, and that $\Pi_{i j}^{h, \mathrm{k}}$ are normalized probability densities, we easily get, to leading order,

$$
\int \mathcal{J}_{1}^{*}\left(f_{1}, f_{2}, \varphi_{3}^{(2)}\right)(\mathbf{v}) d \mathbf{v}=2\left(\nu_{31}^{d} n_{1}+\nu_{32}^{d} n_{2}\right) n_{3}^{(2)},
$$

where $n_{3}^{(2)}$ stands for the number density of the distribution $\varphi_{3}^{(2)}$ given in (4.12). Analogous results are in order for the other chemical contributions:

$$
\begin{aligned}
& \int \mathcal{J}_{1}^{d}\left(f_{1}, f_{2}\right)(\mathbf{v}) d \mathbf{v}=2\left(\nu_{12}^{d} n_{1}+\nu_{22}^{d} n_{2}\right) n_{2}, \\
& \int \mathcal{J}_{1}^{r}\left(f_{1}\right)(\mathbf{v}) d \mathbf{v}=-2 \nu_{11}^{r}\left(n_{1}\right)^{2}, \\
& \int \mathcal{I}_{1}^{*}\left(\varphi_{3}^{(2)}, f_{0}\right)(\mathbf{v}) d \mathbf{v}=2 \nu_{30}^{d} n_{3}^{(2)} n_{0}, \\
& \int \mathcal{I}_{1}^{d}\left(f_{2}, f_{0}\right)(\mathbf{v}) d \mathbf{v}=2 \nu_{20}^{d} n_{2} n_{0} .
\end{aligned}
$$

By inserting results (4.17)-(4.20) into the macroscopic equation (4.16), and then passing to the limit $\epsilon \rightarrow 0$, we obtain the following evolution equation of reaction-diffusion type for the number density $n_{1}$ :

$$
\begin{aligned}
& \frac{\partial n_{1}}{\partial t}-\frac{m_{1}+m_{0}}{2 m_{1} m_{0}} \frac{T_{0}}{\bar{\nu}_{10} n_{0}} \Delta_{\mathbf{x}} n_{1}= \\
& =\frac{2\left(\nu_{30}^{d} n_{0}+\nu_{31}^{d} n_{1}+\nu_{32}^{d} n_{2}\right) \nu_{11}^{r}\left(n_{1}\right)^{2}}{\nu_{30}^{t} n_{0}+\nu_{31}^{t} n_{1}+\nu_{32}^{t} n_{2}}+2\left(\nu_{20}^{d} n_{0}+\nu_{21}^{d} n_{1}+\nu_{22}^{d} n_{2}\right) n_{2}-2 \nu_{11}^{r}\left(n_{1}\right)^{2} .
\end{aligned}
$$

The same procedure may then be applied to the second kinetic equation in (4.1). We realize again that the dominant linear contribution in the momentum equation is 
proportional to the momentum of the distribution $f_{2}$ :

$$
\begin{aligned}
\int \mathbf{v} \mathcal{Q}_{20}\left(f_{2}, f_{0}\right)(\mathbf{v}) d \mathbf{v} & =-\frac{2 m_{0}}{2 m_{1}+m_{0}} \bar{\nu}_{20} \iint(\mathbf{v}-\mathbf{w}) f_{2}(\mathbf{v}) f_{0}(\mathbf{w}) d \mathbf{v} d \mathbf{w}= \\
& =-\frac{2 m_{0}}{2 m_{1}+m_{0}} \bar{\nu}_{20} n_{0} \int \mathbf{v} f_{2}(\mathbf{v}) d \mathbf{v}
\end{aligned}
$$

and, as shown above, this allows to achieve the closure of the streaming part of the continuity equation for $n_{2}$. The relevant scattering contributions now can be cast as

$$
\begin{aligned}
& \int \mathcal{J}_{2}^{*}\left(f_{1}, f_{2}, \varphi_{3}^{(2)}\right)(\mathbf{v}) d \mathbf{v}=\left(\nu_{13}^{i} n_{1}+\nu_{23}^{i} n_{2}\right) n_{3}^{(2)}, \\
& \int \mathcal{J}_{2}^{d}\left(f_{1}, f_{2}\right)(\mathbf{v}) d \mathbf{v}=-\left(\nu_{21}^{d} n_{1}+\nu_{22}^{d} n_{2}\right) n_{2}, \\
& \int \mathcal{I}_{2}^{*}\left(\varphi_{3}^{(2)}, f_{0}\right)(\mathbf{v}) d \mathbf{v}=\nu_{30}^{i} n_{3}^{(2)} n_{0}, \\
& \int \mathcal{I}_{2}^{d}\left(f_{2}, f_{0}\right)(\mathbf{v}) d \mathbf{v}=-\nu_{20}^{d} n_{2} n_{0},
\end{aligned}
$$

so that, in conclusion, the closed reaction-diffusion system for the number densities $n_{1}$ and $n_{2}$ reads as

$$
\begin{aligned}
& \frac{\partial n_{1}}{\partial t}-\frac{m_{1}+m_{0}}{2 m_{1} m_{0}} \frac{T_{0}}{\bar{\nu}_{10} n_{0}} \Delta_{\mathbf{x}} n_{1}= \\
& =\frac{2\left(\nu_{30}^{d} n_{0}+\nu_{31}^{d} n_{1}+\nu_{32}^{d} n_{2}\right) \nu_{11}^{r}\left(n_{1}\right)^{2}}{\nu_{30}^{t} n_{0}+\nu_{31}^{t} n_{1}+\nu_{32}^{t} n_{2}}+2\left(\nu_{20}^{d} n_{0}+\nu_{21}^{d} n_{1}+\nu_{22}^{d} n_{2}\right) n_{2}-2 \nu_{11}^{r}\left(n_{1}\right)^{2} \\
& \frac{\partial n_{2}}{\partial t}-\frac{2 m_{1}+m_{0}}{4 m_{1} m_{0}} \frac{T_{0}}{\bar{\nu}_{20} n_{0}} \Delta_{\mathbf{x}} n_{2}= \\
& =\frac{\left(\nu_{30}^{i} n_{0}+\nu_{31}^{i} n_{1}+\nu_{32}^{i} n_{2}\right) \nu_{11}^{r}\left(n_{1}\right)^{2}}{\nu_{30}^{t} n_{0}+\nu_{31}^{t} n_{1}+\nu_{32}^{t} n_{2}}-\left(\nu_{20}^{d} n_{0}+\nu_{21}^{d} n_{1}+\nu_{22}^{d} n_{2}\right) n_{2} .
\end{aligned}
$$

It is easily checked that this set of partial differential equations obviously fulfils mass conservation, in the sense that the chemical source term for atoms (the right hand side of the first equation) is just twice the opposite of the corresponding chemical term for molecules. This property follows immediately from the fact that $\nu_{3 j}^{t}=\nu_{3 j}^{d}+\nu_{3 j}^{i}$, which is the present version of (4.9). In our Maxwell molecules frame the diffusion coefficients, namely the positive factors in front of the Laplacian operators

$$
D_{i}=\frac{m_{i}+m_{0}}{2 m_{i} m_{0}} \frac{T_{0}}{\bar{\nu}_{i 0} n_{0}}, \quad i=1,2,
$$

turn out to be proportional to the background temperature and inversely proportional to the macroscopic scattering collision frequency with the medium.

\section{Conclusions}

We may notice that reactive contributions in (4.24) are strongly affected by the number density $n_{3}^{(2)}$ (whose expression (4.12) is used in the first term on the right hand sides). This is what in the literature [16] is usually referred to as "ghost effect", 
in the sense that even if the species 3 (unstable molecules) disappears (up to the first order accuracy in the parameter $\epsilon$ ), it leaves an actual trace in the hydrodynamic equations for $n_{1}, n_{2}$; in fact, in the present scaling, chemical operators involving the distribution $\varphi_{3}=O\left(\epsilon^{2}\right)$ are assumed much faster $(O(1 / \epsilon))$ than other reactions $(O(\epsilon))$, so that when passing to the limit $\epsilon \rightarrow 0$ they provide non-vanishing contributions, at the same leading order as the other reactive terms.

The right hand sides in (4.24), denoted by $\mathcal{R}_{1}$ and $\mathcal{R}_{2}$ respectively, fulfill the obvious condition

$$
\mathcal{R}_{1}+2 \mathcal{R}_{2}=0
$$

which reproduces the preservation of total number of atoms, either free or bound together in a molecule. The overall reaction-diffusion system may be rewritten as

$$
\frac{\partial n_{i}}{\partial t}-D_{i} \Delta_{\mathbf{x}} n_{i}=\mathcal{R}_{i}, \quad i=1,2
$$

with diffusion coefficients given by (4.25), and reactive terms defined by (5.1) and by

$$
\mathcal{R}_{2}=-\left(\nu_{20}^{d} n_{0}+\nu_{21}^{d} n_{1}+\nu_{22}^{d} n_{2}\right) n_{2}+\frac{\nu_{30}^{i} n_{0}+\nu_{31}^{i} n_{1}+\nu_{32}^{i} n_{2}}{\nu_{30}^{t} n_{0}+\nu_{31}^{t} n_{1}+\nu_{32}^{t} n_{2}} \nu_{11}^{r}\left(n_{1}\right)^{2} .
$$

The chemical source $\mathcal{R}_{2}$ for stable molecules is then made up by a loss contribution, due to their global dissociation reaction rate, and by a gain contribution, proportional to the recombination reaction rate $\nu_{11}^{r}\left(n_{1}\right)^{2}$ through a reduction factor representing the overall probability of inelastic scattering in collisions relevant to unstable molecules. Such a factor is the only parameter affected by the excited species, via the microscopic collision frequencies $\nu_{3 j}^{k}$. In the considered physical situation, reactive terms $\mathcal{R}_{i}$ are rational functions of the number densities $n_{i}$. Collision equilibria for the fluid-dynamic equations (corresponding to vanishing right hand sides in (5.2)) are given by the requirement

$$
\begin{aligned}
& \nu_{11}^{r}\left(n_{1}\right)^{2}\left(\nu_{30}^{i} n_{0}+\nu_{31}^{i} n_{1}+\nu_{32}^{i} n_{2}\right) \\
& -\left(\nu_{20}^{d} n_{0}+\nu_{21}^{d} n_{1}+\nu_{22}^{d} n_{2}\right)\left(\nu_{30}^{t} n_{0}+\nu_{31}^{t} n_{1}+\nu_{32}^{t} n_{2}\right) n_{2}=0 .
\end{aligned}
$$

Notice that this is a cubic equation for the unknown field $n_{1}$, of the form

$$
A\left(n_{1}\right)^{3}+B\left(n_{1}\right)^{2}-C n_{1}-D=0
$$

with coefficients depending on $n_{2}$, and with $A, C, D>0$. Hence, similarly to [11], just on the basis of the sign of the coefficients, it can be easily checked that there exists a unique admissible (i.e. positive) solution for $n_{1}$ as a function of $n_{2}$. In fact, the other two roots are either real and negative, or complex conjugate with negative real part. Equation (5.4) plays thus the role of a mass action law for chemical equilibrium.

Under different scalings for chemical reactions, we could obtain simpler collision contributions in the macroscopic reaction-diffusion equations for $n_{1}, n_{2}$. Roughly speaking, if in (4.24) the background density $n_{0}$ were assumed much greater than species densities $n_{1}, n_{2}$ (ratio $O\left(1 / \epsilon^{2}\right.$ ) at the dimensional level), or, equivalently, if physical parameters are such that all chemical collision frequencies of the type $\nu_{i 0}^{\mathrm{k}}$ are much greater $(O(1 / \epsilon))$ than the remaining $\nu_{i j}^{k}$ with $j>0$, then only the contributions proportional to $\nu_{20}^{d} n_{0} n_{2}$ would be preserved to the leading order. The relevant 
reaction-diffusion equations would take a completely linear form, and they could be hopefully studied from a mathematical point of view following the same steps outlined in the paper [1]. In this particular case the equilibrium condition would be trivially, but consistently, $n_{2}=0$, that means disappearance also of stable molecules. This drastic regime is avoided if we keep additionally the recombination collision frequency $\nu_{11}^{r}$ greater by a factor $1 / \epsilon$ than the other ones, so that $\mathcal{R}_{2}=\nu_{11}^{r}\left(\nu_{30}^{i} / \nu_{30}^{t}\right)\left(n_{1}\right)^{2}-\nu_{20}^{d} n_{0} n_{2}$, with a non-trivial collision equilibrium, and only a quadratic nonlinearity.

Acknowledgement. This work was performed in the frame of the activities sponsored by MIUR (Project "Mathematical Problems of Kinetic Theories"), by INDAM, by GNFM, and by the University of Parma (Italy), and by the European Network HYKE "Hyperbolic and kinetic equations: Asymptotics, Numerics, Analysis". Helpful discussions with L. Desvillettes are gratefully acknowledged.

\section{REFERENCES}

[1] M. Bisi and L. Desvillettes, From reactive Boltzmann equations to reaction-diffusion systems, J. Stat. Phys., 124, 881-912, 2006.

[2] M. Bisi and G. Spiga, Fluid-dynamic equations for granular particles in a host medium, J. Math. Phys., 46, 113301, 2005.

[3] V. C. Boffi, V. Protopopescu and G. Spiga, On the equivalence between the probabilistic, kinetic, and scattering kernel formulations of the Boltzmann equation, Physica A, 164, 400-410, 1990.

[4] C. Cercignani, The Boltzmann Equation and its Applications, Springer, New York, 1988.

[5] C. Cercignani, Rarefied Gas Dynamics: from Basic Concepts to Actual Calculations, Cambridge University Press, Cambridge, 2000.

[6] F. Conforto, R. Monaco, F. Schürrer and I. Ziegler, Steady detonation waves via the Boltzmann equation for a reacting mixture, J. Phys. A: Math. Gen., 36, 5381-5398, 2003.

[7] A. De Masi, P. A. Ferrari and J. L. Lebowitz, Reaction-diffusion equations for interacting particle systems, J. Statist. Phys., 44, 589-644, 1986.

[8] L. Desvillettes, R. Monaco and F. Salvarani, A kinetic model allowing to obtain the energy law of polytropic gases in the presence of chemical reactions, Eur. J. Mech. B Fluids, 24, 219-236, 2005.

[9] V. Giovangigli, Multicomponent Flow Modeling, Birkhäuser, Boston, 1999.

[10] M. Groppi and J. Polewczak, On two kinetic models for chemical reactions: comparisons and existence results, J. Statist. Phys., 117, 211-241, 2004.

[11] M. Groppi, A. Rossani and G. Spiga, Kinetic theory of a diatomic gas with reactions of dissociation and recombination through a transition state, J. Phys. A, 33, 8819-8833, 2000.

[12] G. M. Kremer, M. Pandolfi Bianchi and A. J. Soares, A relaxation kinetic model for transport phenomena in a reactive flow, Phys. Fluids, 18, 037104, 2006.

[13] I. Müller and T. Ruggeri, Extended Thermodynamics, Springer, New York, 1993.

[14] A. Rossani and G. Spiga, A note on the kinetic theory of chemically reacting gases, Physica A, 272, 563-573, 1999.

[15] B. D. Shizgal and A. Chikhaoui, On the use of temperature parametrized rate coefficients in the estimation of non-equilibrium reaction rates, Physica A, 365, 317-332, 2006.

[16] Y. Sone, Kinetic Theory and Fluid Dynamics, Birkhäuser, Boston, 2002.

[17] R. Spigler and D. H. Zanette, Asymptotic analysis and reaction-diffusion approximation for BGK kinetic models of chemical processes in multispecies gas mixtures, J. Appl. Math. Phys. (ZAMP), 44, 812-827, 1993.

[18] Y. Yoshizawa, Wave structures of a chemically reacting gas by the kinetic theory of gases, Potter J. L. (Ed.), Rarefied Gas Dynamics, A. I. A. A., New York, 501-517, 1977.

[19] D. H. Zanette, Linear and nonlinear diffusion and reaction-diffusion equations from discretevelocity kinetic models, J. Phys. A: Math. Gen., 26, 5339-5349, 1993.

[20] F. Zus and H. Olivier, A kinetic model for chemical reactions in hypersonic non-equilibrium flows, Proceedings of GAMM 2005, Proc. Appl. Math. Mech. 5, 533-534, 2005. 